\title{
Effectiveness of CPPI Strategies under Discrete--Time Trading
}

\author{
Sven Balder \\ Michael Brandl \\ Antje Mahayni \\ Department of Banking and Finance \\ University of Bonn
}

This version: October 5, 2005

\begin{abstract}
.
The paper analyzes the effectiveness of the constant proportion portfolio insurance (CPPI) method under trading restrictions. If the CPPI method is applied in continuous time, the CPPI strategies provide a value above a floor level unless the price dynamic of the risky asset permits jumps. The risk of violating the floor protection is called gap risk. In practice, it is caused by liquidity constraints and price jumps. Both can be modelled in a setup where the price dynamic of the risky asset is described by a continuous-time stochastic process but trading is restricted to discrete time. We propose a discrete-time version of the continuous-time CPPI strategies which satisfies three conditions. The resulting strategies are self-financing, the asset exposure is non-negative and the value process converges. We determine risk measures such as the shortfall probability and the expected shortfall and discuss criteria which ensure that the gap risk does not increase to a level which contradicts the original intention of portfolio insurance.
\end{abstract}

JEL: G11, G12

Keywords: Portfolio insurance, discrete-time trading, return guarantees, gap risk, volatility risk.

\footnotetext{
${ }^{1}$ Corresponding Author: Antje Mahayni, Department of Banking and Finance, University of Bonn, Adenauerallee 24-42, D-53113 Bonn, Germany, phone: +49 (228) 736103, fax: +49 (228) 735050, e-mail: antje.mahayni@uni-bonn.de

The authors would like to thank Klaus Sandmann for helpful discussions.
} 


\title{
EFFECTIVENESS OF CPPI STRATEGIES UNDER DISCRETE-TIME TRADING
}

\begin{abstract}
The paper analyzes the effectiveness of the constant proportion portfolio insurance (CPPI) method under trading restrictions. If the CPPI method is applied in continuous time, the CPPI strategies provide a value above a floor level unless the price dynamic of the risky asset permits jumps. The risk of violating the floor protection is called gap risk. In practice, it is caused by liquidity constraints and price jumps. Both can be modelled in a setup where the price dynamic of the risky asset is described by a continuous-time stochastic process but trading is restricted to discrete time. We propose a discrete-time version of the continuous-time CPPI strategies which satisfies three conditions. The resulting strategies are self-financing, the asset exposure is nonnegative and the value process converges. We determine risk measures such as the shortfall probability and the expected shortfall and discuss criteria which ensure that the gap risk does not increase to a level which contradicts the original intention of portfolio insurance.
\end{abstract}

\section{INTRODUCTION}

Financial strategies which are designed to limit downside risk and at the same time to profit from rising markets are summarized in the class of portfolio insurance strategies. Among others, Grossman and Villa (1989) and Basak (2002) define a portfolio insurance trading strategy as a strategy which guarantees a minimum level of wealth at a specified time horizon, but also participates in the potential gains of a reference portfolio. The most prominent examples of dynamic versions are the constant proportion portfolio insurance (CPPI) strategies and option-based portfolio insurance (OBPI) strategies with synthetic puts. ${ }^{1}$ Here, synthetic is understood in the sense of a trading strategy in basic (traded) assets which creates the put. In a complete financial market model, there exists a perfect hedge, i.e. a self-financing and duplicating strategy. In contrast, the introduction of market incompleteness impedes the concept of perfect hedging.

In this paper, the incompleteness is caused by trading restrictions. The price process of the benchmark index, i.e. the risky asset, is driven by a continuous-time process, while trading is restricted to discrete time. Therefore, the effectiveness of the OBPI approach is given by the effectiveness of a discrete-time option hedge. The error of time-discretizing a continuous-time hedging strategy for a put (or call) is extensively studied in the literature. Discretely adjusted option hedges, first analyzed in Boyle and Emanuel (1980), are also treated in Bertsimas, Kogan, and Lo (1998) and more recently in Mahayni (2003),

\footnotetext{
${ }^{1}$ Option-based portfolio insurance (OBPI) with synthetic puts is introduced in Leland and Rubinstein (1976), constant proportion portfolio insurance (CPPI) in Black and Jones (1987). For the basic procedure of the CPPI see also Merton (1971).
} 
Talay and Zheng (2003) and Hayashi and Mykland (2005). ${ }^{2}$ While the implications of discrete-time option hedges for portfolio protection are interesting in themselves, the main focus of this paper is on the effects of time-discretizing the CPPI strategies which has, to our knowledge, not been done yet. However, we keep in mind that the OBPI is one alternative to the CPPI.

The optimality of an investment strategy depends on the risk profile of the investor. In order to determine the optimal rule, one has to solve for the strategy which maximizes the expected utility. Thus, portfolio insurers can be modelled by utility maximizers where the maximization problem is given under the additional constraint that the value of the strategy is above a specified wealth level. Without postulating completeness, we refer to the works of Cox and Huang (1989), Brennan and Schwartz (1989), Grossman and Villa (1989), Grossman and Zhou (1993, 1996), Basak (1995), Cvitanic and Karatzas (1995, 1999), Browne (1999), Tepla $(2000,2001)$ and El Karoui, Jeanblanc, and Lacoste (2005). Mostly, the solution of the maximization problem is given by the unconstrained problem including a put option. Obviously, this is in the spirit of the OPBI method. The introduction of various sources of market incompleteness in terms of stochastic volatility and trading restrictions makes the determination of an optimal investment rule under minimum wealth constraints quite difficult if not impossible. For example, if the payoff of a put (or call) option is not attainable, the OBPI approach is not a viable method in the above setup. ${ }^{3}$ Another problem is posed by model risk. This is generated by the possible inconsistency between the unknown true model and the model the risk manager relies on in order to determine the hedging strategy. That is, one has to use some (educated) assumptions about the data-generating processes. However, strategies which are based on an optimality criterion with respect to one particular model, fail to be optimal if the "true" asset price dynamics deviate from the assumed ones. Summing up, one alternative to the maximization approach, either based on utility or other optimality criteria, is given by a more general analysis of robustness properties of a stylized strategy. ${ }^{4}$

For the reasons given above, we follow an approach where the analysis is already based on stylized portfolio strategies, i.e. we take the CPPI rule as given. Because of its simplicity and the possibility to customize it to the preferences of an investor, the CPPI has become very popular with practitioners.

In Black and Perold (1992), it is shown that in a complete market, the CPPI can be characterized as expected utility maximizing when the utility function is piecewise HARA and the guaranteed level is growing with the riskless interest rate. Obviously, this argument loses it validity if an additional incompleteness is introduced by trading restrictions. The properties of continuous-time CPPI strategies are studied extensively in the literature, c.f. Bookstaber and Langsam (2000) or Black and Perold (1992). A comparison of OBPI

\footnotetext{
${ }^{2}$ Transaction costs can naturally explain the reason for discrete-time hedging. The implication of transaction costs, conducted by Leland (1985), is studied in Bensaid, Lesne, and Scheinkman (1992), Boyle and Vorst (1992), Avellaneda and Parás (1994), Grannan and Swindle (1996) and Toft (1996).

${ }^{3}$ Hedging strategies in incomplete markets depend on some dynamic risk measure that has to be minimized. For a discussion, see e.g. Schweizer (2001).

${ }^{4}$ With respect to the robustness of option hedges we refer the reader to Avellaneda, Levy, and Parás (1995), Lyons (1995), Bergman, Grundy, and Wiener (1996), El Karoui, Jeanblanc-Picqué, and Shreve (1998), Hobson (1998), Dudenhausen, Schlögl, and Schlögl (1998) and Mahayni (2003).
} 
and CPPI (in continuous time) is given in Bertrand and Prigent (2002a). An empirical investigation of both methods can, for example, be found in Do (2002) who simulates the performance of these strategies using Australian data. The literature also deals with the effects of jump processes, stochastic volatility models and extreme value approaches on the CPPI method, c.f. Bertrand and Prigent (2002b), Bertrand and Prigent (2003). In contrast to this, we consider the risk resulting from trading restrictions. We propose a discrete-time version of a simple CPPI strategy which satisfies three conditions. The strategy is self-financing, the asset exposure is non-negative and the value process converges. Assuming that the underlying price process is given by a geometric Brownian motion, trading restrictions in the sense of discrete-time trading are sufficient to model the possibility of a floor violation. The advantage of a model setup along the lines of Black and Scholes (1973) is that risk measures, such as the shortfall probability and the expected shortfall which are implied by the discrete-time CPPI method can be given in closed form. It is shown that the same holds for the price of the gap risk. Of course, this is only possible because of the proportional structure of the CPPI strategies. A CPPI investor specifies two parameters, a constant multiplier and the floor (or guarantee). Then the amount which is invested in a risky asset is determined by the product of the multiplier and the excess of the portfolio value over the floor. The remaining part, i.e. the difference of the portfolio value and the asset exposure is invested in a riskless asset. This implies that the strategy is self-financing. If the price process of the risky asset does not permit jumps, the continuous-time application of the CPPI ensures that the portfolio value does not fall below the floor. The strategy outperforms the prescribed floor unless there is a sudden drop in market prices such that the investor is not able to rebalance his portfolio adequately. With respect to trading restrictions, the effectiveness of the discrete-time strategies should improve with an increasing trading frequency which, of course, is also true for a discrete-time option hedge. However, a synthetic put can only be represented by a stochastic multiplier. Intuitively, this explains why the risk measures can be given in closed form in the case of a discrete-time CPPI but not in the case of a discrete-time option based strategy.

In our setup, once the risk measures are determined, the gap risk can be priced easily. However, the main focus is not the pricing. Instead, the relevant risk measures are used to discuss criteria which must be satisfied such that the CPPI strategy is still effective if applied in discrete time. ${ }^{5}$ For example, it turns out that for a small number of rehedges, the shortfall probability, i.e. the probability that the strategy falls below the floor at the terminal date, may as well first increase in the trading frequency before it decreases. However, after a critical number of rehedges, the shortfall probability is always decreasing in the number of rehedges. The change in monotonicity can be interpreted in terms of a minimal number of rehedges which is necessary such that a portfolio protection can be achieved by applying the CPPI technique in discrete time. Obviously, the critical number of rehedges depends on the model parameters, the drift and the volatility, and the strategy parameters, in particular the multiplier. The same is true for the risk measures. We discuss criteria which ensure that the CPPI method is effective in a discrete-time

\footnotetext{
${ }^{5}$ It is worth mentioning that while arbitrage free pricing is based on the expectation under the martingale measure, the risk measures must be determined with respect to the real world measure.
} 
setup. If the volatility is known, it is, for example, possible to specify the strategy parameters of a CPPI, i.e. the multiplier and the floor, such that the shortfall probability is bounded above by a confidence level. The effects of model and strategy parameters on the risk measures are illustrated by examples.

The outline of the paper is as follows. Sec. 2 gives the model setup, motivates the CPPI method and reviews the structure and the properties of continuous-time CPPI strategies. A discrete-time version of a CPPI strategy where the asset exposure is restricted to be non-negative is defined in Sec. 3. The properties of the discrete-time version are derived in analogy to the continuous-time version. The assumption that the asset price increments are independent and identically distributed yields a closed-form solution for the shortfall probability and the expected shortfall. The calculations are given in Sec. 4 which also includes a sensitivity analysis of the risk measures with respect to model and strategy parameters. Besides, it is shown that the value process of the discrete-time version converges to the value process of the continuous-time strategy in distribution if the trading restrictions vanish. Sec. 5 illustrates the results and discusses criteria which ensure that the discrete-time strategy is effective, i.e. the portfolio protection is still valid in discrete time. Sec. 6 concludes the paper.

\section{Model Setup}

All stochastic processes are defined on a stochastic basis $\left(\Omega, \mathcal{F},\left(\mathcal{F}_{t}\right)_{t \in\left[0, T^{*}\right]}, P\right)$ which satisfies the usual hypotheses.

We consider two investment possibilities: a risky asset $S$ and a riskless bond $B$ which grows with constant interest rate $r$, i.e. $d B_{t}=B_{t} r d t$ where $B_{0}=b$. The evolution of the risky asset $S$, a stock or benchmark index, is given by a geometric Brownian motion, i.e.

$$
d S_{t}=S_{t}\left(\mu d t+\sigma d W_{t}\right), \quad S_{0}=s,
$$

where $W=\left(W_{t}\right)_{0 \leq t \leq T}$ denotes a standard Brownian motion with respect to the real world measure $P$. $\mu$ and $\sigma$ are constants and we assume that $\mu>r \geq 0$ and $\sigma>$ 0 . A continuous-time investment strategy or saving plan for the interval $[0, T]$ can be represented by a predictable process $\left(\alpha_{t}\right)_{0 \leq t \leq T}$. $\alpha_{t}$ denotes the fraction of the portfolio value at time $t$ which is invested in the risky asset $S$. If there are no additional borrowing restrictions, we can, w.l.o.g., restrict ourselves to strategies which are self-financing, i.e. strategies where money is neither injected nor withdrawn during the trading period $] 0, T[$. Thus, the amount which is invested at date $t$ in the riskless bond $B$ is given in terms of the fraction $1-\alpha_{t} . V=\left(V_{t}\right)_{0 \leq t \leq T}$ denotes the portfolio value process which is associated with the strategy $\alpha$, i.e. $V_{t}$ is the solution of

$$
d V_{t}(\alpha)=V_{t}\left(\alpha_{t} \frac{d S_{t}}{S_{t}}+\left(1-\alpha_{t}\right) \frac{d B_{t}}{B_{t}}\right), \text { where } V_{0}=x .
$$

Notice that there are alternative possibilities for portfolio insurance. Let $T$ denote the terminal trading date. For example, one might think of $T$ as the retirement day. The minimal wealth which must be obtained is denoted by $G$. The guaranteed amount is assumed to be less than the terminal value of a pure bond investment, i.e. we assume $G<e^{r T} V_{0}$. Besides a pure bond investment, a trivial possibility is given by a static trading strategy where at the initial time $t=0$ the present value of the guarantee, i.e. 
$G e^{-r T}$ is invested in the bond $B$ and the remaining part, i.e. the surplus $V_{0}-e^{-r T} G$, is invested in the risky asset $S$. Thus, although $\alpha_{t}=\frac{\left(V_{0}-e^{-r T} G\right)}{V_{t}} \frac{S_{t}}{S_{0}}$ is stochastic, the strategy is static in the sense that there are no rebalancing decisions involved during the interval ]0,T]. Abstracting from stochastic interest rates, the above strategy honors the guarantee $G$ independent of the stochastic process generating the asset prices. Another example of portfolio insurance is given by a stop-loss-strategy which is represented by a portfolio fraction $\alpha_{t}=1_{\left\{V_{t}>e^{-r(T-t)} G\right\}}$. Here, everything is invested in the asset until the cushion (or surplus) $V_{t}-e^{-r(T-t)} G$ is exhausted. This means that the strategy is effective with respect to the guarantee if continuous-time monitoring (trading) is possible and the asset price process does not permit jumps. Together, the above strategies can be used to explain the basic idea of the constant proportion portfolio insurance. A combination of continuous-time monitoring and keeping the cushion under control yields the CPPI approach.

However, in a complete market there is a second possibility, the option based portfolio insurance approach. The completeness implies that there is a self-financing and duplicating strategy in $S$ and $B$ for any claim with payoff $h\left(S_{T}\right)$ at $T$. Notice that for $h\left(S_{T}\right)=\lambda\left(S_{T}+\left[\frac{G}{\lambda}-S_{T}\right]^{+}\right)=G+\lambda\left[S_{T}-\frac{G}{\lambda}\right]^{+}$and $\lambda>0$ it holds $h\left(S_{T}\right) \geq G$. Buying $\lambda$ assets and $\lambda$ put-options with strike $\frac{G}{\lambda}$ enables a portfolio insurance, too. ${ }^{6}$ If the associated options are not traded, they must be synthesized by a hedging strategy in $S$ and $B$. If the concept of perfect hedging is impeded by market incompleteness, the OBPI and the CPPI can both violate the purpose of portfolio insurance. In terms of model risk, i.e. the problem that one does not know which process can describe the true data generating process adequately, the OBPI approach causes more problems than the CPPI technique. The composition of the CPPI strategy is model independent. In contrast to this, it is necessary to incorporate a volatility guess in order to implement the OBPI approach with synthetic options. Thus, there is an additional error introduced by using the wrong hedging model.

In the following, we concentrate on the CPPI approach. It is worth mentioning that even without an utility based justification, the CPPI is an important strategy in practice. ${ }^{7}$ We fix the notation and review the basic form and properties of continuous-time CPPI strategies. Recall that the basic idea of the CPPI approach is to invest the amount of portfolio value which is above the present value of the guarantee in the risky asset $S$. Normally, the symbol $F$ is used to denote the present value of the guarantee $G$, i.e. $F_{t}:=\exp \{-r(T-t)\} G$. This is equivalent to

$$
d F_{t}=F_{t} r d t \quad \text { with } F_{0}=\exp \{-r T\} G .
$$

The surplus is called cushion $C$, i.e. $C_{t}:=V_{t}-F_{t}$. If the cushion is monitored in continuous time, it is even possible to invest a multiple of the cushion in the risky asset.

\footnotetext{
${ }^{6}$ Or buying $\lambda$ call options with strike $\frac{G}{\lambda}$ and a riskless investment of $G e^{-r T}$.

${ }^{7}$ Besides the importance of CPPI strategies in the context of hedge funds, the CPPI technique has recently been extended to the credit derivatives market, c.f. Fletcher (2005). ABN Amro created the first credit CPPI product in April 2004. It is called Rente Booster.
} 
Let $m$ denote the multiplier, then the fraction $\alpha$ of a CPPI strategy is given by ${ }^{8}$

$$
\alpha_{t}:=\frac{m C_{t}}{V_{t}}
$$

Notice that there are various extensions to the CPPI. For example, besides borrowing constraints it is also possible to permit only a maximal fraction of wealth to be invested in the risky asset. Furthermore, one might think of a floor adjustment which allows to protect the gains in the case of a favorable asset performance. However, all these extensions prohibit closed-form solutions. For this reason, we call a continuous-time CPPI strategy which satisfies the above form simple. Notice that a simple CPPI-strategy is given in terms of the guarantee $G$ and the multiplier $m \geq 0$. Normally, a CPPI even implies that $m \geq 2$. In addition to the protection feature this ensures that the value of the CPPI strategy is convex in the asset price, at least in a continuous-time setup with continuous asset paths. Throughout the paper, the guarantee is given exogenously, i.e. it is the minimal value of wealth which is needed at $T$. In contrast to the OBPI approach, the CPPI includes an additional degree of freedom which is introduced by the multiplier $m$. While CPPI strategies are protective with respect to the guarantee for all $m \geq 0$, this is not true if trading is restricted to discrete time. Heuristically, this is easily explained by the static case where a protection is only possible for $m \leq 1$.

For the sake of completeness, we review some basic properties of the continuous-time CPPI technique. First, consider the cushion process $\left(C_{t}\right)_{0 \leq t \leq T}$.

LEMmA 2.1. If the asset price dynamic is lognormal, i.e. if it satisfies Equation (1), the cushion process $\left(C_{t}\right)_{0 \leq t \leq T}$ of a simple CPPI is lognormal, too. In particular, it holds

$$
d C_{t}=C_{t}\left(\left(r+m(\mu-r) d t+\sigma m d W_{t}\right) .\right.
$$

Proof: Notice that $C_{t}:=V_{t}-F_{t}$ implies

$$
\begin{aligned}
d C_{t} & =d\left(V_{t}-F_{t}\right) \\
& =V_{t}\left(\frac{m C_{t}}{V_{t}} \frac{d S_{t}}{S_{t}}+\left(1-\frac{m C_{t}}{V_{t}}\right) \frac{d B_{t}}{B_{t}}\right)-F_{t} \frac{d B_{t}}{B_{t}} \\
& =C_{t}\left(m \frac{d S_{t}}{S_{t}}-(m-1) r d t\right) .
\end{aligned}
$$

The rest of the proof follows with Equation (1).

Proposition 2.2. The $t$-value of the a simple CPPI with parameter $m$ and $G$ is

$$
V_{t}=G e^{-r(T-t)}+\frac{V_{0}-G e^{-r T}}{S_{0}^{m}} \exp \left\{\left(r-m\left(r-\frac{1}{2} \sigma^{2}\right)-m^{2} \frac{\sigma^{2}}{2}\right) t\right\} S_{t}^{m} .
$$

Proof: Notice that Equation (3) can also be represented as follows

$$
V_{t}=F_{t}+\frac{V_{0}-F_{0}}{S_{0}^{m}} \exp \left\{\left(r-m\left(r-\frac{1}{2} \sigma^{2}\right)-m^{2} \frac{\sigma^{2}}{2}\right) t\right\} S_{t}^{m} .
$$

\footnotetext{
${ }^{8}$ For simplicity, we abstract from borrowing constraints which can be modelled by $\alpha_{t}=$ $\frac{\min \left\{m\left(V_{t}-F_{t}\right), p V_{t}\right\}}{V_{t}}$ with $p \geq 0$.
} 
The proof of this equation is well known, c.f. for example Bertrand and Prigent (2002a). Together with

$$
S_{t}=S_{0} e^{\left(\mu-\frac{1}{2} \sigma^{2}\right) t+\sigma W_{t}}
$$

it follows that

$$
V_{t}-F_{t}=\left(V_{0}-F_{0}\right) e^{\left(r+m(\mu-r)-\frac{1}{2} m^{2} \sigma^{2}\right) t+\sigma m W_{t}}
$$

which matches the result of Lemma 2.1.

Equation (3) illustrates the basic property of a simple CPPI. The $t$-value of the strategy consists of the present value of the guarantee $G$, i.e. the floor at $t$, and a non-negative part which is proportional to $\left(\frac{S_{t}}{S_{0}}\right)^{m}$. Thus, the value process of a simple CPPI strategy is path independent. ${ }^{9}$ The payoff above the guarantee is linear for $m=1$ and it is convex for $m \geq 2$. In financial terms, the payoff of a CPPI strategy with $m \geq 2$ can be interpreted as a power claim. The portfolio protection is efficient with probability one, i.e. the terminal value of the strategy is higher than the guarantee with probability one.

The expected value and the variance of a simple CPPI are easily calculated as follows.

LEMMA 2.3.

$$
\begin{aligned}
E\left[V_{t}\right] & =F_{t}+\left(V_{0}-F_{0}\right) \exp \{(r+m(\mu-r)) t\} \\
\operatorname{Var}\left[V_{t}\right] & =\left(V_{0}-F_{0}\right)^{2} \exp \{2(r+m(\mu-r)) t\}\left(\exp \left\{m^{2} \sigma^{2} t\right\}-1\right) .
\end{aligned}
$$

Proof: With Equation (4) it follows

$$
\begin{aligned}
E\left[\ln \left(V_{t}-F_{t}\right)\right] & =\ln \left(V_{0}-F_{0}\right)+\left(r+m(\mu-r)-\frac{1}{2} m^{2} \sigma^{2}\right) t \\
\operatorname{Var}\left[\ln \left(V_{t}-F_{t}\right)\right] & =\sigma^{2} m^{2} t .
\end{aligned}
$$

Notice that for $X \sim N\left(\mu_{X}, \sigma_{X}\right)$ we have

$$
E\left[e^{X}\right]=e^{\mu_{X}+\frac{1}{2} \sigma_{X}^{2}}, \quad \operatorname{Var}\left[e^{X}\right]=e^{2 \mu_{X}} e^{\sigma_{X}^{2}}\left(e^{\sigma_{X}^{2}}-1\right) .
$$

It is worth mentioning that the expected terminal value of a simple CPPI strategy is independent of the volatility $\sigma$. In contrast, the standard deviation increases exponentially in the volatility of the asset $S$, c.f. Figure 1 and 2. Intuitively, this property explains that the effectiveness of a CPPI strategy with respect to various sources of market incompleteness does not only depend on the asset price drift but even more importantly on the volatility of the underlying asset. In particular, this is the case for a rather high value of the multiplier.

\footnotetext{
${ }^{9}$ Notice that this is not true if one deviates from the concept of a simple CPPI.
} 


\section{Expectation and Standard Deviation of a simple CPPI}

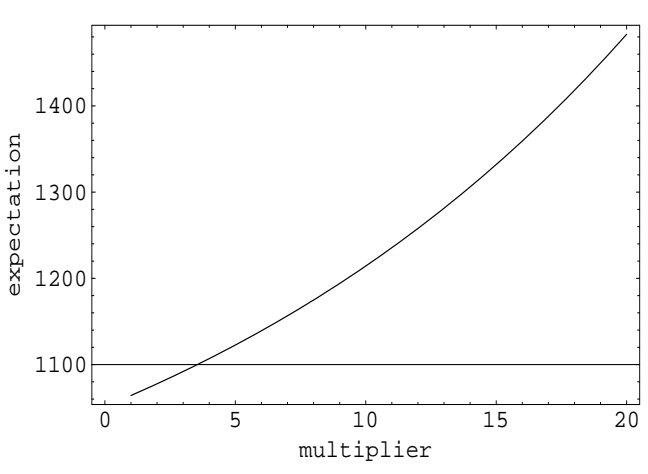

Figure 1. Expected final value of a simple CPPI with $V_{0}=1000, G=800, T=1$ and varying $m$ for $\sigma=0.1$, $\mu=0.1$ and $r=0.05$.

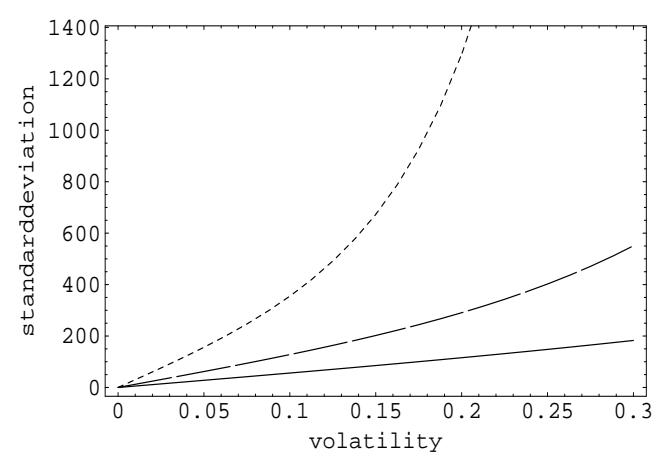

FiguRE 2. Standard deviation of the final value of a simple CPPI with $V_{0}=1000, G=$ $800, T=1$ and varying $\sigma$ for $\mu=0.1, r=0.05$ and $m=2$ ( $m=4, m=8$ respectively).

\section{TRADING RESTRICTIONS}

We assume now that trading is restricted to a discrete set of dates and define a discretetime version of the simple CPPI strategy satisfying the following three conditions. Firstly, the value process of the discrete-time version converges in distribution to the value process of the continuous-time simple CPPI strategy. Secondly, the discrete-time version is a self-financing strategy. This means, that after the initial investment $V_{0}=x$, there are no in- or outflow of funds. Thirdly, the strategy does not allow for a negative asset exposure. Notice that the first condition implies that the cushion process of the discrete-time version converges to a lognormal process in distribution. However, the cushion process with respect to a discrete-time set of trading dates may also be negative. Therefore, to avoid a negative asset exposure, this must be captured by the definition of the discrete-time version.

Let $\tau^{n}$ denote a sequence of equidistant refinements of the interval $[0, T]$, i.e.

$$
\tau^{n}=\left\{t_{0}^{n}=0<t_{1}^{n}<\cdots<t_{n-1}^{n}<t_{n}^{n}=T\right\}
$$

where $t_{k+1}^{n}-t_{k}^{n}=\frac{T}{n}$ for $k=0, \cdots, n-1$. To simplify the notation, we drop the superscript $n$ and denote the set of trading dates with $\tau$ instead of $\tau^{n}$. The restriction that trading is only possible immediately after $t_{k} \in \tau$ implies that the number of shares held in the risky asset is constant on the intervals $\left.] t_{i}, t_{i+1}\right]$ for $i=0, \ldots, n-1$. However, the fractions of wealth which are invested in the assets change as assets prices fluctuate. Thus, it is necessary to consider the number of shares held in the risky asset $\eta$ and the number of bonds $\beta$, i.e. the tupel $\phi=(\eta, \beta)$. With respect to the continuous-time simple CPPI 
strategies, it holds

$$
\begin{aligned}
\eta_{t} & =\frac{\alpha_{t} V_{t}}{S_{t}}=\frac{m C_{t}}{S_{t}} \\
\beta_{t} & =\frac{\left(1-\alpha_{t}\right) V_{t}}{B_{t}}=\frac{V_{t}-m C_{t}}{B_{t}} .
\end{aligned}
$$

The following argumentation illustrates that a time-discretized strategy $\phi^{\tau}$ which is defined by

$$
\left.\left.\phi_{t}^{\tau}:=\phi_{t_{k}} \text { for } t \in\right] t_{k}, t_{k+1}\right], k=0, \ldots, n-1
$$

is in general not self-financing. The value process $V^{\tau}:=V(\phi ; \tau)$ which is associated with the discrete-time version of $\phi$, i.e. with $\phi^{\tau}$, is defined by $V_{0}^{\tau}:=V_{0}$ and

$$
\begin{aligned}
V_{t}(\phi ; \tau) & \left.\left.:=\eta_{t_{k}} S_{t}+\beta_{t_{k}} B_{t} \quad \text { for } t \in\right] t_{k}, t_{k+1}\right] \\
& \left.\left.=V_{t}(\phi)-\left(\eta_{t}-\eta_{t_{k}}\right) S_{t}-\left(\beta_{t}-\beta_{t_{k}}\right) B_{t} \quad \text { for } t \in\right] t_{k}, t_{k+1}\right],
\end{aligned}
$$

where

$$
V_{t}(\phi):=\eta_{t} S_{t}+\beta_{t} B_{t}
$$

If $\phi$ is self-financing, this is not necessarily true for $\phi^{\tau}$. Notice that $\phi^{\tau}$ is self-financing iff

$$
\begin{aligned}
\eta_{t_{k}} S_{t_{k+1}}+\beta_{t_{k}} B_{t_{k+1}} & =\eta_{t_{k+1}} S_{t_{k+1}}+\beta_{t_{k+1}} B_{t_{k+1}} \quad \text { for all } k=0, \ldots, n-1 \\
\Longleftrightarrow V_{t_{k+1}}(\phi ; \tau) & =V_{t_{k+1}}(\phi) \text { for all } k=0, \ldots, n-1 .
\end{aligned}
$$

Obviously, this is only true in the limit, i.e. for $n \rightarrow \infty$. It is worth mentioning that it is not even clear whether the above time-discretized version is mean-self-financing with respect to the real world measure, c.f. for example Mahayni (2003). In order to specify a meaningful discrete-time version of a simple CPPI strategy, it is necessary to admit only self--financing strategies. This is equal to the condition that

$$
\left.\left.\beta_{t}^{\tau}=\frac{1}{B_{t_{k}}}\left(V_{t_{k}}^{\tau}-\eta_{t}^{\tau} S_{t_{k}}\right) \quad \text { for } t \in\right] t_{k}, t_{k+1}\right]
$$

Finally, recall that constant proportion portfolio insurance means that the fraction of wealth $\alpha$ which is invested in the risky asset is given proportionally to the difference of the portfolio value and the floor, i.e. the cushion. Let $C^{\tau}$ denote the discrete-time version of the cushion process $C$, then

$$
C_{t}^{\tau}:=V_{t}^{\tau}-F_{t}
$$

In addition, we do not allow for short positions in the risky asset, i.e. the asset exposure is bounded below by zero. Thus, it is necessary to consider the positive part of the cushion. The above reasoning gives the following definition.

Definition 3.1 (Discrete-Time CPPI). A strategy $\phi^{\tau}=\left(\eta^{\tau}, \beta^{\tau}\right)$ where for $\left.\left.t \in\right] t_{k}, t_{k+1}\right]$ and $k=0, \ldots, n-1$

$$
\begin{aligned}
\eta_{t}^{\tau} & :=\max \left\{\frac{m C_{t_{k}}^{\tau}}{S_{t_{k}}}, 0\right\} \\
\beta_{t}^{\tau} & :=\frac{1}{B_{t_{k}}}\left(V_{t_{k}}^{\tau}-\eta_{t}^{\tau} S_{t_{k}}\right)
\end{aligned}
$$


is called simple discrete-time CPPI.

Proposition 3.2 (Discrete-time cushion process). Define

$$
t_{s}:=\min \left\{t_{k} \in \tau \mid V_{t_{k}}^{\tau}(\alpha)-F_{t_{k}} \leq 0\right\}
$$

with $t_{s}=\infty$ if the minimum is not attained. It holds

$$
V_{t_{k+1}}^{\tau}-F_{t_{k+1}}=e^{r\left(t_{k+1}-\min \left\{t_{s}, t_{k+1}\right\}\right)}\left(V_{t_{0}}^{\tau}-F_{t_{0}}\right) \prod_{i=1}^{\min \{s, k+1\}}\left(m \frac{S_{t_{i}}}{S_{t_{i-1}}}-(m-1) e^{r \frac{T}{n}}\right) .
$$

Proof: Notice that

$$
\begin{aligned}
V_{t_{k+1}}^{\tau} & =\max \left\{\frac{m C_{t_{k}}^{\tau}}{S_{t_{k}}}, 0\right\} S_{t_{k+1}}+\left(V_{t_{k}}^{\tau}-\max \left\{\frac{m C_{t_{k}}^{\tau}}{S_{t_{k}}}, 0\right\} S_{t_{k}}\right) \frac{B_{t_{k+1}}}{B_{t_{k}}} \\
& = \begin{cases}F_{t_{k}} \frac{B_{t_{k+1}}}{B_{t_{k}}}+\left(V_{t_{k}}^{\tau}-F_{t_{k}}\right)\left(m \frac{S_{t_{k+1}}}{S_{t_{k}}}-(m-1) \frac{B_{t_{k+1}}}{B_{t_{k}}}\right) & \text { for } V_{t_{k}}^{\tau}-F_{t_{k}}>0 \\
V_{t_{k}}^{\tau} \frac{B_{t_{k+1}}}{B_{t_{k}}} & \text { for } V_{t_{k}}^{\tau}-F_{t_{k}} \leq 0 .\end{cases}
\end{aligned}
$$

Together with $F_{t_{k}} \frac{B_{t_{k+1}}}{B_{t_{k}}}=F_{t_{k+1}}$ it follows

$$
V_{t_{k+1}}^{\tau}-F_{t_{k+1}}= \begin{cases}\left(V_{t_{k}}^{\tau}-F_{t_{k}}\right)\left(m \frac{S_{t_{k+1}}}{S_{t_{k}}}-(m-1) e^{r \frac{T}{n}}\right) & \text { for } V_{t_{k}}^{\tau}-F_{t_{k}}>0 \\ \left(V_{t_{k}}^{\tau}-F_{t_{k}}\right) e^{r \frac{T}{n}} & \text { for } V_{t_{k}}^{\tau}-F_{t_{k}} \leq 0\end{cases}
$$

for all $k=0, \ldots, n-1$. In particular, we have

$$
V_{T}^{\tau}= \begin{cases}V_{t_{s}}^{\tau} e^{r\left(T-t_{s}\right)} & \text { for } t_{s} \leq t_{n-1} \\ G_{T}+\left(V_{t_{n-1}}^{\tau}-F_{t_{n-1}}\right)\left(m \frac{S_{t_{n}}}{S_{t_{n-1}}}-(m-1) \frac{B_{t_{n}}}{B_{t_{n-1}}}\right) & \text { for } t_{s} \geq t_{n}\end{cases}
$$

Notice that the value process $V^{\tau}$ converges in distribution to the value process $V$ if the trading restrictions vanish, i.e. if $n \rightarrow \infty$. The proof is based on the convergence of the corresponding expectation and variance. Therefore, it is postponed to the end of the following section where we calculate the moments and risk measures of the discrete-time CPPI.

\section{Risk Measures of Discrete-Time CPPI}

Recall that the basic idea of a CPPI strategy is a portfolio protection. Heuristically, the usage of these strategies is explained by an investor who wants to participate in bullish markets but does not want the terminal value of the strategy to end up below a guaranteed amount $G$. Thus, the investor is completely risk averse for values below the floor (or guarantee). As motivated in the previous sections, as soon as a source of market incompleteness is considered, i.e. a restriction on the set of trading dates, the concept of a perfect portfolio protection is impeded, in particular for dynamic strategies. With the exception of static portfolio insurance strategies, there is a positive probability that the terminal value is below the guaranteed amount. In particular, this is true for CPPI and OBPI strategies which include a synthetic put. The use of such constrained strategies or strategies which include a gap risk can be explained as follows. On the one hand, one might think of an investor who accepts, because of market incompleteness, a strategy which gives the guaranteed amount with a certain success probability. On the other hand, 
one might think of retail products which are based on the CPPI method and are thus also hedged by a CPPI strategy. Normally, the buyer of such a product gets the guaranteed amount even in the case that the strategy fails to provide it. Here, the issuer takes the gap risk and considers this in his product pricing. In both cases, the risk profile of the CPPI is of great interest. It is necessary to compute risk measures which allow a characterization if the constrained CPPI is still effective in terms of portfolio insurance.

In the following, we take the view of an investor who uses the CPPI as a savings plan with portfolio protection. A CPPI strategy contradicts the original idea of the portfolio insurance if it results in a very high gap risk, i.e. if the shortfall probability and the expected shortfall are prohibitively high. The investor has to decide whether this additional risk is not too high in terms of a portfolio insurance. In addition to the expected final value and its standard deviation, we consider the shortfall probability and the expected shortfall given default as the risk measures which determine the effectiveness of the discrete-time CPPI strategy. ${ }^{10}$ The shortfall probability is the probability that the final value of the discrete-time CPPI strategy is less or equal to the guaranteed amount $G$. Intuitively, one can also define a local shortfall probability (given that no prior shortfall happened before). Additionally, we use the expected shortfall given default to describe the amount which is lost if a shortfall occurs.

DEFinition 4.1 (Risk measures).

$$
\begin{aligned}
P^{S F} & :=P\left(V_{T}^{\tau} \leq G\right)=P\left(V_{T}^{\tau} \leq F_{T}\right) & & \text { shortfall probability } \\
P_{t_{i}, t_{i+1}}^{L S F} & :=P\left(V_{t_{i+1}}^{\tau} \leq F_{t_{i+1}} \mid V_{t_{i}}^{\tau}>F_{t_{i}}\right) & & \text { local shortfall probability } \\
E S & := & E\left[G-V_{T}^{\tau} \mid V_{T}^{\tau} \leq G\right] & \text { expected shortfall given default. }
\end{aligned}
$$

It turns out that, in contrast to a discrete-time option based strategy with synthetic put, the calculation of the shortfall probability implied by a CPPI strategy is very simple. This is easily explained if one observes that the shortfall event is equivalent to the event that the stopping time which is defined in Proposition 3.2 is prior to the terminal date. It is convenient to consider the following lemma.

LEMmA 4.2. Let $A_{k}:=\left\{\frac{S_{t_{k}}}{S_{t_{k-1}}}>\frac{m-1}{m} e^{r \frac{T}{n}}\right\}$ for $k=1, \ldots, n$, then it holds

$$
\left\{t_{s}>t_{i}\right\}=\bigcap_{j=1}^{i} A_{j} \text { and }\left\{t_{s}=t_{i}\right\}=A_{i}^{C} \cap\left(\bigcap_{j=1}^{i-1} A_{j}\right) \quad \text { for } i=1, \ldots, n \text {. }
$$

\footnotetext{
${ }^{10}$ Notice that the shortfall probability is not a coherent risk measure, i.e. it is not sub-additive. In contrast, the expected shortfall given default is a coherent risk measure. We remain within the class of stylized strategies, i.e. the CPPI strategies. Thus, it is in fact not a problem even if the effectiveness of the strategies is analyzed by using a risk measure which is not sub-additive. For details on coherent risk measures we refer to the work of Artzner, Delbaen, Eber, and Heath (1999).
} 


\section{Shortfall probability}

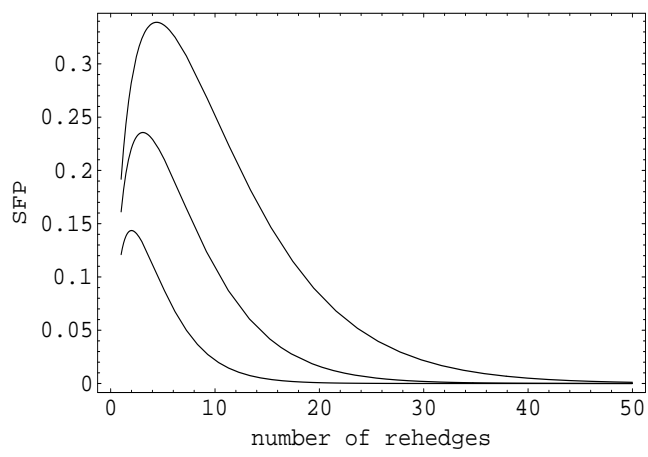

Figure $3 . \quad V_{0}=1000, G_{T}=$ $1000, m=12$ (15 and 18 respectively), $\mu=0.085, r=$ 0.05 and $\sigma=0.1$.

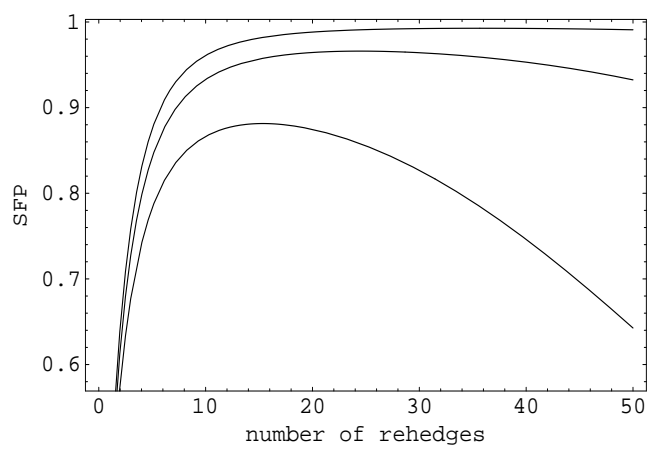

Figure 4. $V_{0}=1000, G_{T}=$ $1000, m=12$ (15 and 18 respectively), $\mu=0.085, r=$ 0.05 and $\sigma=0.3$.

Proof: According to the poof of Proposition 3.2 it holds

$$
V_{t_{k+1}}^{\tau}-F_{t_{k+1}}= \begin{cases}\left(V_{t_{k}}^{\tau}-F_{t_{k}}\right)\left(m \frac{S_{t_{k+1}}}{S_{t_{k}}}-(m-1) e^{r \frac{T}{n}}\right) & \text { for } V_{t_{k}}^{\tau}-F_{t_{k}}>0 \\ \left(V_{t_{k}}^{\tau}-F_{t_{k}}\right) e^{r \frac{T}{n}} & \text { for } V_{t_{k}}^{\tau}-F_{t_{k}} \leq 0 .\end{cases}
$$

The rest of the proof follows immediately with the definition of the stopping time $t_{s}$ and

$$
m \frac{S_{t_{k+1}}}{S_{t_{k}}}-(m-1) e^{r \frac{T}{n}}>0 \Longleftrightarrow \frac{S_{t_{k+1}}}{S_{t_{k}}}>\frac{m-1}{m} e^{r \frac{T}{n}} .
$$

LEMMA 4.3. The local shortfall probability is independent of $t_{i}$ and $t_{i+1}$, i.e.

$$
\begin{aligned}
P_{t_{i}, t_{i+1}}^{L S F}=P^{L S F} & =\mathcal{N}\left(-d_{2}\right) \\
\text { where } d_{2} & :=\frac{\ln \frac{m}{m-1}+(\mu-r) \frac{T}{n}-\frac{1}{2} \sigma^{2} \frac{T}{n}}{\sigma \sqrt{\frac{T}{n}}} .
\end{aligned}
$$

Proof: Notice that

$$
P_{t_{i}, t_{i+1}}^{\mathrm{LSF}}=P\left(V_{t_{i+1}}^{\tau} \leq F_{t_{i+1}} \mid V_{t_{i}}^{\tau}>F_{t_{i}}\right)=P\left(t_{s}=t_{i+1} \mid t_{s}>t_{i}\right)=P\left(\frac{S_{t_{1}}}{S_{t_{0}}} \leq \frac{m-1}{m} e^{r \frac{T}{n}}\right),
$$

where the last equality follows with Lemma 4.2 and the assumption that the asset price increments are independent and identically distributed (iid).

Proposition 4.4. The shortfall probability $P^{S F}$ is given in terms of the local shortfall probability $P^{L S F}$, i.e.

$$
P^{S F}=1-\left(1-P^{L S F}\right)^{n} .
$$

Proof: The above lemma is a direct consequence of Lemma 4.2 and the independence of asset price increments.

$$
P^{\mathrm{SF}}=1-P\left(t_{s}=\infty\right)=1-\left(1-P^{\mathrm{LSF}}\right)^{n} .
$$


It can be shown, c.f. Lemma C.1 of the appendix, that the shortfall probability converges to zero if we approach continuous-time trading, i.e. $\lim _{n \rightarrow \infty} P^{\mathrm{SF}}=0$. At first glance, it might be tempting to think that the shortfall probability is monotonically decreasing in the hedging frequency, i.e. the number of rehedges $n$. In general, this is only true after a sufficiently high $n$ is reached. The effect that the shortfall probability is increasing for small $n$ is more pronounced for high volatilities and high multipliers, c.f. Figure 3 and Figure $4 .{ }^{11}$ Let $n^{*}$ denote the number of rehedges such that the shortfall probability is increasing in $n$ for $n \leq n^{*}$ and decreasing for $n \geq n^{*}$. The critical level $n^{*}$ is to be interpreted as a minimal number of rehedges which is necessary such that the CPPI method is effective for $m \geq 2$ in discrete time. Consider for example a guaranteed amount $G$ given by $G=e^{r T \frac{m}{m}-1} V_{0}$ such that $\alpha_{0}=1$, i.e. the initial exposure in the risky asset coincides with the initial investment. If in addition $n$ is chosen to be one, i.e. there is no rehedge until $T$, the discrete-time CPPI strategy coincides with a pure asset investment. Obviously, the CPPI method can not be effective for $n=1$, i.e. a pure asset investment is not in the spirit of the CPPI method. Thus, it is intuitively clear that a minimal number of rehedges becomes necessary such that the CPPI method applies if trading is restricted to discrete time. The critical level $n^{*}$ and its implications are further discussed in Sec. 5 where the effectiveness of the discrete-time CPPI method is studied in detail.

If a shortfall is possible, one should also consider the amount of the shortfall or a risk measure which describes the amount of the shortfall. One possibility is given by the expected shortfall ES which is introduced in Definition 4.1. It turns out that in order to determine the expected shortfall, it is convenient to decompose the expected terminal payoff into two parts. One part is given by the expected terminal value if a shortfall occurs and the other by the expectation on the set where the terminal value is above the guarantee.

Proposition 4.5 (Expected final value). It holds

$$
\begin{aligned}
E\left[V_{T}^{\tau}\right] & =G+\left(V_{0}-F_{0}\right)\left[E_{1}^{n}+e^{-r \frac{T}{n}} E_{2} \frac{e^{r T}-E_{1}^{n}}{1-E_{1} e^{-r \frac{T}{n}}}\right] \\
\text { where } E_{1} & :=m e^{\mu \frac{T}{n}} \mathcal{N}\left(d_{1}\right)-e^{r \frac{T}{n}}(m-1) \mathcal{N}\left(d_{2}\right) \\
E_{2} & :=e^{r \frac{T}{n}}\left[1+m\left(e^{(\mu-r) \frac{T}{n}}-1\right)\right]-E_{1} .
\end{aligned}
$$

$d_{2}$ is the same as in Lemma 4.3 and $d_{1}:=d_{2}+\sigma \sqrt{\frac{T}{n}}$.

Proof: Notice that

$$
E\left[V_{T}^{\tau}\right]=E\left[V_{T}^{\tau} 1_{\left\{t_{s}=\infty\right\}}\right]+E\left[\begin{array}{ll}
V_{T}^{\tau} & \left.1_{\left\{t_{s} \leq t_{n}\right\}}\right]
\end{array} .\right.
$$

With Lemma 4.2 and Lemma A.1 of Appendix A it follows

$$
\begin{aligned}
E\left[V_{T}^{\tau} 1_{\left\{t_{s}=\infty\right\}}\right] & =E\left[F_{T} \prod_{i=1}^{n} 1_{\left\{\frac{S_{t_{i}}}{S_{t_{i-1}}}>\frac{m-1}{m} e^{r \frac{T}{n}}\right\}}\right]+E\left[\left(V_{T}^{\tau}-F_{T}\right) \prod_{i=1}^{n} 1_{\left\{\frac{S_{t_{i}}}{S_{t_{i-1}}}>\frac{m-1}{m} e^{r \frac{T}{n}}\right\}}\right] \\
& =G P\left(t_{s}=\infty\right)+\left(V_{0}-F_{0}\right) E_{1}^{n}=G\left(1-P^{\mathrm{SF}}\right)+\left(V_{0}-F_{0}\right) E_{1}^{n} .
\end{aligned}
$$

\footnotetext{
${ }^{11}$ It is straightforward to show that the shortfall probability is monotonically increasing in $m$ and $\sigma$.
} 
For the second expectation, observe that

$$
E\left[V_{T}^{\tau} 1_{\left\{t_{s} \leq t_{n}\right\}}\right]=\sum_{i=1}^{n} E\left[V_{T}^{\tau} 1_{\left\{t_{s}=t_{i}\right\}}\right] .
$$

The remaining part of the proof follows with Lemma A.2 of Appendix A and

$$
\begin{aligned}
& \sum_{i=1}^{n} e^{r\left(T-t_{i}\right)}\left(F_{t_{i}} P^{\mathrm{LSF}}\left(1-P^{\mathrm{LSF}}\right)^{i-1}+E_{2} E_{1}^{i-1}\left(V_{0}-F_{0}\right)\right) \\
= & G P^{\mathrm{SF}}+\left(V_{0}-F_{0}\right) e^{-r \frac{T}{n}} E_{2} \frac{e^{r T}-E_{1}^{n}}{1-E_{1} e^{-r \frac{T}{n}}} .
\end{aligned}
$$

The calculation of the expected shortfall ES is now straightforward. ${ }^{12}$

COROLlary 4.6 (Expected Shortfall). The expected shortfall ES which is defined as in Definition 4.1 is given by

$$
E S=G-\frac{\left(V_{0}-F_{0}\right) e^{-r \frac{T}{n}} E_{2} \frac{e^{r T}-E_{1}^{n}}{1-E_{1} e^{-r \frac{T}{n}}}}{P^{S F}} .
$$

Proof: According to the definition, it holds

$$
\mathrm{ES}=E\left[G-V_{T}^{\tau} \mid t_{s}<\infty\right]=G-\frac{E\left[V_{T}^{\tau} 1_{\left\{t_{s} \leq t_{n}\right\}}\right]}{P^{S F}} .
$$

The proof is completed by inserting the result given in the proof of Proposition 4.5.

Proposition 4.7 (Variance of final value). It holds

$$
\begin{aligned}
& \operatorname{Var}\left[V_{T}^{\tau}\right]=\left(V_{0}-F_{0}\right)^{2}\left[\widetilde{E}_{1}^{n}+e^{-2 r \frac{T}{n}} \widetilde{E}_{2} \frac{e^{2 r T}-\widetilde{E}_{1}^{n}}{1-e^{-2 r \frac{T}{n}} \tilde{E}_{1}}\right]-\left(E\left[V_{T}^{\tau}\right]-G\right)^{2} \\
& \text { where } \\
& \begin{array}{l}
\widetilde{E}_{1}:=m^{2} e^{\left(2 \mu+\sigma^{2}\right) \frac{T}{n}} \mathcal{N}\left(d_{3}\right)-2 m(m-1) e^{(\mu+r) \frac{T}{n}} \mathcal{N}\left(d_{1}\right)+(m-1)^{2} e^{2 r \frac{T}{n}} \mathcal{N}\left(d_{2}\right), \\
\widetilde{E}_{2}:=m^{2} e^{\left(2 \mu+\sigma^{2}\right) \frac{T}{n}}-2 m(m-1) e^{(\mu+r) \frac{T}{n}}+(m-1)^{2} e^{2 r \frac{T}{n}}-\widetilde{E}_{1} .
\end{array}
\end{aligned}
$$

$d_{1}, d_{2}$ are defined as above and

$$
d_{3}:=\frac{2 \ln \frac{m}{m-1}+2(\mu-r) \frac{T}{n}+3 \sigma^{2} \frac{T}{n}}{4 \sigma \sqrt{\frac{T}{n}}} .
$$

Proof: Notice that

$$
\operatorname{Var}\left[V_{T}^{\tau}\right]=\operatorname{Var}\left[V_{T}^{\tau}-F_{T}\right]=E\left[\left(V_{T}^{\tau}-F_{T}\right)^{2}\right]-\left(E\left[V_{T}^{\tau}\right]-F_{T}\right)^{2}
$$

where $E\left[\left(V_{T}^{\tau}-F_{T}\right)^{2}\right]=E\left[\left(V_{T}^{\tau}-F_{T}\right)^{2} 1_{\left\{t_{s}=\infty\right\}}\right]+\sum_{i=1}^{n} E\left[\left(V_{T}^{\tau}-F_{T}\right)^{2} 1_{\left\{t_{s}=t_{i}\right\}}\right]$.

\footnotetext{
${ }^{12}$ The same is true for the price of the associated gap risk, i.e. the price of an option where the payoff at $T$ is given by $\left(G-V_{T}^{\tau}\right)^{+}$. Notice that by standard financial theory, the $t_{0}$-price is given by the expected value of the discounted payoff under the martingale measure. However, the risk measures which are considered here must be given with respect to the real world measure.
} 


\section{Sensitivity of risk measures}

\begin{tabular}{|c|c|c|}
\hline Risk measures & Strategy parameter & Model parameter \\
\hline & $m$ & $\mu$ \\
\hline Mean & $\uparrow$ & $\uparrow$ \\
\hline Stdv. & $\downarrow$ & $\uparrow$ \\
\hline$P^{\mathrm{SF}}$ & 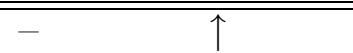 & $\bar{l} \downarrow$ \\
\hline$E S F$ & $\downarrow$ & $\uparrow$ \\
\hline
\end{tabular}

TABLE 1. Sensitivity analysis of risk measures. We use the symbol $\uparrow$ for monotonically increasing and $\downarrow$ for monotonically decreasing.

Analogously to the proof of Proposition 4.5, it follows with Lemma B.1 and Lemma B.2 of the appendix that

$$
E\left[\left(V_{T}^{\tau}-F_{T}\right)^{2}\right]=\left(V_{0}-F_{0}\right)^{2} \widetilde{E}_{1}^{n}+\sum_{i=1}^{n}\left(V_{0}-F_{0}\right)^{2} e^{2 r\left(T-t_{i}\right)} \widetilde{E}_{2} \widetilde{E}_{1}^{i-1} .
$$

The remaining part of the proof follows with

$$
\sum_{i=1}^{n} e^{2 r\left(T-t_{i}\right)} \widetilde{E}_{1}^{i-1}=e^{-2 r \frac{T}{n}} \frac{e^{2 r T}-\widetilde{E}_{1}^{n}}{1-e^{-2 r \frac{T}{n}} \tilde{E}_{1}} .
$$

The calculation of the expectation and variance of the discrete-time CPPI strategy can now be used to prove the convergence, i.e.

Proposition 4.8 (Convergence). For $n \rightarrow \infty$, the value process $V^{\tau}$ converges to the value process $V$ in distribution, i.e.

$$
V^{\tau} \stackrel{\mathcal{L}}{\rightarrow} V
$$

In particular, it holds

$$
\begin{aligned}
\lim _{n \rightarrow \infty} E\left[V_{T}^{\tau}\right] & =G+\left(V_{0}-F_{0}\right) \exp \{(r+m(\mu-r)) T\} \\
\lim _{n \rightarrow \infty} \operatorname{Var}\left[V_{T}^{\tau}\right] & =\left(V_{0}-F_{0}\right)^{2} \exp \{2(r+m(\mu-r)) T\}\left(\exp \left\{m^{2} \sigma^{2} T\right\}-1\right) .
\end{aligned}
$$

Proof: The proof is given in Appendix C.

Before we study the effectiveness of the time-discretized CPPI in detail, we end this section with a sensitivity analysis of the risk measures. In order to avoid a lengthy discussion of all possible sensitivities, we summarize the main results in Table 1. The corresponding proofs are straightforward. Notice that the shortfall probability is independent of $G$, c.f. Proposition 4.4. Partial differentiation immediately yields that the shortfall probability is increasing in $\sigma$ and $m$ but decreasing in $\mu$. In contrast, the sensitivity analysis of the other risk measures is tedious. For example, the monotonicity of the expected terminal value, i.e. $E\left[V_{T}^{\tau}\right]$, in $\sigma$ is shown in Appendix D. Similar arguments to the ones presented here can also be used to show that the expected terminal payoff is also increasing in $\mu$ and $m$. Monotonicity in $G$ and $V$ is immanent. With respect to the standard deviation, it is intuitively clear that the the volatility $\sigma$ has a positive effect on the standard deviation, so does $m$. It is worth mentioning that both the shortfall probability and the expected 


\section{Moments of continuous-time CPPI}

\begin{tabular}{|c|c|c|}
\hline multiplier $m$ & Mean & Stdv. Dev. \\
\hline 12 & $1078.03(1078.03)$ & $140.04(1387.90)$ \\
15 & $1086.67(1086.67)$ & $252.51(7801.45)$ \\
18 & $1096.27(1096.27)$ & $476.83(62763.30)$ \\
\hline
\end{tabular}

TABLE 2. Moments.

shortfall are increasing in $m$ and $\sigma$. This implies that a discrete-time CPPI is not effective in discrete time if either the standard deviation is too high in comparison to the multiplier or vice versa.

\section{Effectiveness of the DiscRete-time CPPI Method}

As shown above, the effectiveness of the discrete-time CPPI method depends on the strategy parameters, i.e. the multiplier $m$, the number of rehedges $n$ and the guarantee $G$, as well as the model parameters $\mu$ and $\sigma$. The most important influences are caused by the multiplier $m$ and the volatility $\sigma$. Therefore, all examples are considered for varying multipliers and volatilities. If not mentioned otherwise, we consider a model scenario where $\mu=0.085, \sigma=0.1$ ( 0.2 or 0.3 , respectively) and $r=0.05$. The time to maturity of the CPPI strategy is equal to one year $(T=1)$, the initial investment coincides with the guarantee, i.e. $V_{0}=G=1000$. Thus, the goal of the strategies under consideration is to ensure $100 \%$ of the initial capital. This is in accordance to guaranteed fund management. ${ }^{13}$ For the multiplier $m$ we consider the values 12,15 and 18 . Here, the initial asset exposure $m\left(V_{0}-e^{-r T} G\right)$ is 585.247 for $m=12,731.559$ for $m=15$ and 877.870 for $m=18$ such that the relative initial asset investment varies between 0.585 and 0.88 . A high multiplier is convenient in order to emphasize all effects and to highlight the effect of a small change in volatility.

First, we consider the question whether the discrete-time CPPI method gives a good approximation of the continuous-time CPPI for a finite number of rehedges $n$. Recall that the value process of the discrete-time CPPI converges to the value process of the continuous-time CPPI in distribution, c.f. Proposition 4.8. Since the cushion process of the continuous-time CPPI is lognormal, the payoff distribution of the continuous-time CPPI is described by its mean and its standard deviation. These numbers are summarized in Table 2. In comparison, Table 3 summarizes the moments and risk measures for various numbers of rehedges $n$.

Now consider the shortfall probability. Observe, that in the case where $\sigma=0.1$, a monthly CPPI-strategy $(n=12)$ with a multiplier $m=12$ implies a shortfall probability of only 0.01 . In contrast, a volatility of $\sigma=0.2$ gives a shortfall probability of more than 0.5 . Thus, the monthly CPPI strategy ensures a significant protection level for $\sigma=0.1$ while

\footnotetext{
${ }^{13}$ It is worth mentioning that the probability that the CPPI portfolio value is higher than the OBPI value increases in the percentage of the insured initial investment, c.f. Bertrand and Prigent (2003). Recall that $V_{T}^{\text {OBPI }}=G+\left[S_{T}-G\right]^{+}$. Thus, the above effect is intuitively explained by observing that the probability of exercising the embedded call option is decreasing in the strike.
} 
Moments of discrete-time CPPI

\begin{tabular}{|c|c|c|c|c|r|}
\hline $\mathrm{n}$ & $m$ & Mean & Stdv. Dev. & SFP & $E S F$ \\
\hline 12 & 12 & $1077.53(1080.23)$ & $125.04(703.03)$ & $\mathbf{0 . 0 1 1 5} \mathbf{( 0 . 5 4 3 0 )}$ & $5.463(25.933)$ \\
24 & 12 & $1077.77(1078.60)$ & $132.01(948.79)$ & $0.0002(0.3195)$ & $2.981(12.296)$ \\
48 & 12 & $1077.90(1077.98)$ & $135.88(1133.36)$ & $0.0000(0.0580)$ & $1.574(5.802)$ \\
96 & 12 & $1077.97(1077.97)$ & $137.92(1249.06)$ & $0.0000(0.0009)$ & $0.000(3.037)$ \\
\hline \hline $\mathrm{n}$ & $m$ & Mean & Stdv. Dev. & $S F P$ & $E S F$ \\
\hline 12 & 15 & $1085.94(1074.28)$ & $206.30(1874.59)$ & $0.0767(0.7592)$ & $8.901(57.01)$ \\
24 & 15 & $1086.22(1090.92)$ & $226.81(3361.17)$ & $0.0069(0.6610)$ & $4.836(27.86)$ \\
48 & 15 & $1086.44(1087.43)$ & $238.86(4936.18)$ & $0.0000(0.3258)$ & $2.597(11.03)$ \\
96 & 15 & $1086.56(1086.60)$ & $245.46(6130.89)$ & $0.0000(0.0333)$ & $1.364(5.02)$ \\
\hline \hline $\mathrm{n}$ & $m$ & Mean & Stdv. Dev. & $S F P$ & $E S F$ \\
\hline 12 & 18 & $1095.70(1120.63)$ & $339.07(4924.65)$ & $0.2094(0.8691)$ & $13.911(118.32)$ \\
24 & 18 & $1095.65(1111.58)$ & $396.37(12759.40)$ & $\mathbf{0 . 0 4 9 4} \mathbf{( 0 . 8 5 9 3 )}$ & $7.296(64.66)$ \\
48 & 18 & $1095.90(1101.08)$ & $432.75(25691.30)$ & $0.0015(0.6767)$ & $3.908(23.70)$ \\
96 & 18 & $1096.08(1096.68)$ & $453.66(39053.60)$ & $0.0000(0.2131)$ & $2.067(8.30)$ \\
\hline \hline
\end{tabular}

TABLE 3. The time horizon is $T=1$ year and the guarantee $G$ is equal to the initial investment $V_{t_{0}}=1000$. The model parameters are given by $\mu=0.085$, $r=0.05$ and $\sigma=0.1$ ( $\sigma=0.2$ respectively).

the concept of portfolio insurance is already impeded for $\sigma=0.2$. Here (for $\sigma=0.2$ ), even a weekly rehedging, i.e. $n=48$ is not enough to achieve a shortfall probability of less than 0.05. This illustrates that the effectiveness of the discrete-time CPPI method is very sensitive to the volatility of the asset price process. Besides, the higher the multiplier, the more pronounced the effect is. For example, notice that the shortfall probability for a CPPI-strategy with $n=24$ and $m=18$ is 0.049 for $\sigma=0.1$ but 0.86 for $\sigma=0.2$.

Recall that the shortfall probability is not necessarily monotonically decreasing in the number of rehedges. A very large shortfall probability implies that the number of rehedges is still too low to achieve an effective portfolio protection. For example, one might think of the extreme case that $n=1$, i.e. the case where the portfolio is held constantly on the trading period $[0, T]$. Obviously, a portfolio protection can only be achieved if only the surplus is invested in the risky asset. One can argue that the CPPI method is not effective if the number of rehedges $n$ is still in a region where the shortfall probability is increasing in $n$. Thus, it is convenient to determine the minimal number $n^{*}$ such that an increase in the number of portfolio rebalancing dates is able to reduce the shortfall probability. For different combinations of $\sigma$ and $m$, the critical number $n^{*}$ is illustrated in Table $4 .{ }^{14}$ However, $n^{*}$ can only be used as a number which is at least necessary to achieve an effective portfolio insurance.

One solution to ensure the effectiveness of the discrete-time CPPI method is given by the possibility to determine the contract parameters such that the probability of falling below the guarantee is bounded above by a confidence level $\gamma$, for example $\gamma=0.99$ (or

\footnotetext{
${ }^{14}$ Compare also the remarks in the last section referring to Figure 3 and Figure 4.
} 
Minimal number of rehedges

\begin{tabular}{|rrr|rrr|rrr|}
\hline$m$ & $\sigma$ & $n^{*}$ & $m$ & $\sigma$ & $n^{*}$ & $m$ & $\sigma$ & $n^{*}$ \\
\hline 12 & 0.1 & 2.00 & 15 & 0.1 & 3.08 & 18 & 0.1 & 4.40 \\
12 & 0.2 & 7.00 & 15 & 0.2 & 11.09 & 18 & 0.2 & 16.11 \\
12 & 0.3 & 15.35 & 15 & 0.3 & 24.44 & 18 & 0.3 & 35.64 \\
\hline
\end{tabular}

TABLE 4. Minimal number $n^{*}$ of rehedges such that the shortfall probability is decreasing in $n$.

$\gamma=0.95)$. This can be explained by an investor who is aware of market incompleteness and accepts a small shortfall probability with respect to the guarantee. Again, we consider the same model scenario where $T=1, \mu=0.085, r=0.05, V_{0}=G=1000$ and distinguish between $\sigma=0.1$ and $\sigma=0.2$. For illustration, we determine $(n, m)$-tupels which give a shortfall probability of 0.01 and 0.05 . The resulting values as well as the corresponding other risk measures are given in Table 5. For example, observe that in the case of $\sigma=0.1$, the CPPI method with monthly rehedging and a multiplier of 11.84 ensures that the capital is maintained with a probability of 0.99. At the same time the expected payoff and the variance of the payoff are similar to the ones obtained by a direct investment in $S$, i.e. for the expectation compare 1077 to 1088 and for the standard deviation compare 121.75 to $109.14{ }^{15}$ Therefore, in the case where $\sigma=0.1$, even a monthly rehedging is enough to give a high success probability if the multiplier is chosen appropriately. ${ }^{16}$ However, in case of a volatility scenario where $\sigma=0.2$, the multiplier is to be chosen much more conservatively. Finally, it is worth mentioning that it is sufficient to control the shortfall probability if one also wants to control the expected shortfall which is unarguably a more convincing risk measure. In the above example, it is approximately the same if one keeps the shortfall probability on a 0.01 level or if one keeps the expected shortfall at a level of 5.2.

\section{CONCLUSION}

The introduction of market incompleteness and model risk impedes the concept of dynamic portfolio insurance, i.e. the technique of constant portfolio insurance. The introduction

\footnotetext{
${ }^{15} \mathrm{~A}$ direct investment of $V_{0}$ in the asset $S$ gives for $\sigma=0.1(\sigma=0.2$ respectively)

$$
\begin{aligned}
E\left[V_{0} \frac{S_{T}}{S_{0}}\right] & =V_{0} e^{\mu T}=1088.72(1088.72) \\
\sqrt{\operatorname{Var}\left[V_{0} \frac{S_{T}}{S_{0}}\right]} & =V_{0} \sqrt{e^{\left(2 \mu+\sigma^{2}\right) T}-e^{2 \mu T}}=109.144(219.939) \\
P\left(V_{0} \frac{S_{T}}{S_{0}} \leq G\right) & =0.212(0.373) .
\end{aligned}
$$
}

${ }^{16}$ Again, it is worth mentioning that although a multiplier of approximately 12 seems to be fairly large, it is to be interpreted in combination with the low volatility. In particular, a multiplier of $m=11.843$ implies that for a guarantee $G=V_{0}=1000$ the initial amount invested in $S$ is given by

$$
\alpha V_{0}=m\left(V_{0}-F_{0}\right)=11.843\left(1000-e^{-0.05} 1000\right)=577.59 \text {. }
$$


Risk profile for discrete-time CPPI strategies with a shortfall probability of $0.01(0,05)$.

\begin{tabular}{|r|r||r|r|r|}
\hline \multicolumn{5}{|c|}{$\sigma=0.1$} \\
\hline$n$ & $m$ & Mean & Stdv. & $E S$ \\
\hline 12 & $11.843(14.124)$ & $1077.118(1083.377)$ & $121.752(178.420)$ & $5.313(7.770)$ \\
24 & $15.446(18.024)$ & $1087.558(1095.730)$ & $246.087(398.225)$ & $5.157(7.319)$ \\
36 & $18.146(20.956)$ & $1096.273(1106.154)$ & $432.362(774.426)$ & $5.149(7.217)$ \\
48 & $20.386(23.389)$ & $1104.150(1115.646)$ & $717.129(1419.070)$ & $5.186(7.219)$ \\
60 & $22.336(25.507)$ & $1111.528(1124.588)$ & $1152.310(2511.390)$ & $5.243(7.267)$ \\
\hline \hline \multicolumn{5}{|c|}{$\sigma=0.2$} \\
\hline$n$ & $m$ & Mean & Stdv. & $E S$ \\
\hline 12 & $6.065(7.152)$ & $1063.302(1065.747)$ & $107.138(150.350)$ & $4.478(6.432)$ \\
24 & $7.879(9.128)$ & $1067.464(1070.485)$ & $204.334(316.650)$ & $4.275(5.931)$ \\
36 & $9.234(10.605)$ & $1070.748(1074.241)$ & $345.136(591.266)$ & $4.190(5.720)$ \\
48 & $10.358(11.829)$ & $1073.591(1077.500)$ & $554.966(1048.690)$ & $4.145(5.605)$ \\
60 & $11.335(12.893)$ & $1076.156(1080.449)$ & $868.650(1804.760)$ & $4.121(5.535)$ \\
\hline \multicolumn{5}{|c|}{ TABLE 5. For a given discretization in terms of $n$, the multiplier is determined } \\
such that the implied shortfall probability is $0.01(0.05$ respectively).
\end{tabular}

of tradings restrictions is one possibility to model a gap risk in the sense that a CPPI strategy can not be adjusted adequately. Measuring the risk that the value of a CPPI strategy is less than the floor (or guaranteed amount) is of practical importance for at least two reasons. On the one hand, CPPI strategies are common in hedge funds and retail products. Often, a CPPI strategy is pre-specified in the term sheet of hedge funds. In addition, it is combined with a guarantee for the investor. Thus, an additional option is written. The option is exercised if the value of the CPPI strategy is below the floor. On the other hand, CPPI strategies can be used to protect return guarantees which are embedded in unit-linked life insurance contracts. The terminal date $T$ is interpreted as the time of retirement and the guarantee is interpreted as the amount which is at least needed by the insured. The assumption that the insurer wants to back up the guarantee by a simple and discrete-time investment strategy highlights some advantages in favor of the CPPI method. Firstly, it is computationally very simple and it can easily be applied in discrete time. Secondly, the composition of a CPPI strategy is independent of the model assumption of the investor or insurer who might use a misspecified model. Thirdly, the riskiness in terms of commonly used risk measures which is induced by trading restrictions can be given in closed form. In particular, this is also true for the price of an additional option which is normally included in CPPI-based products.

The analysis of the risk measures of a discrete-time CPPI strategy poses various problems which are to be considered. Basically, it is necessary to check the associated risk measures and to determine whether the strategy is still effective in terms of portfolio protection. For example, the protection feature is violated if the shortfall probability of the CPPI strategy under consideration exceeds the shortfall probability of a pure asset investment. Formally, the last one can be interpreted as a static CPPI. Intuitively, this explains the 
result that the shortfall probability of a discrete-time CPPI is only decreasing in the hedge frequency after a sufficiently high number of rehedges. Below this critical number, the shortfall probability increases such that additional adjustments of the strategy yield a shortfall probability which is even higher than the one of a pure asset investment. This effect is even more pronounced for high asset price volatilities and high multipliers. Thus, if one restricts the set of admissible strategies to those strategies which satisfy a confidence level of protection, the choice of the CPPI-multiplier is naturally restricted. A similar reasoning is applied to other risk measures such as the expected shortfall.

\section{Appendix A. Proof of Proposition 4.5}

Lemma A.1. Let $d_{1}, d_{2}$ and $E_{1}$ be defined as in Proposition 4.5, then it holds

$$
E\left[\left(V_{t_{i}}^{\tau}-F_{t_{i}}\right) \prod_{j=1}^{i} 1\left\{\frac{S_{t_{j}}}{S_{t_{j-1}}}>\frac{m-1}{m} e^{r \frac{T}{n}}\right\}\right]=\left(V_{0}-F_{0}\right)\left(E_{1}\right)^{i} \quad \text { for all } i=1, \ldots, n \text {. }
$$

Proof: The following calculations are based on Proposition 3.2, Lemma 4.2 and the assumption that the asset price increments are independent and identically distributed (iid).

$$
\begin{aligned}
& E\left[\left(V_{t_{i}}^{\tau}-F_{t_{i}}\right) \prod_{j=1}^{i}\left\{\frac{S_{t_{j}}}{S_{t_{j-1}}} \frac{m-1}{m} e^{r \frac{T}{n}}\right\}\right] \\
= & \left(V_{0}^{\tau}-F_{0}\right) E\left[\prod_{j=1}^{i}\left(m \frac{S_{t_{j}}}{S_{t_{j-1}}}-(m-1) e^{r \frac{T}{n}}\right) 1_{\left.\left\{\frac{S_{t_{j}}}{S_{t_{j-1}}}>\frac{m-1}{m} e^{r \frac{T}{n}}\right\}\right]}\right] \\
= & \left(V_{0}^{\tau}-F_{0}\right) \prod_{j=1}^{i} E\left[\left(m \frac{S_{t_{j}}}{S_{t_{j-1}}}-(m-1) e^{r \frac{T}{n}}\right) 1_{\left.\left\{\frac{S_{t_{j}}}{S_{t_{j-1}}}>\frac{m-1}{m} e^{r \frac{T}{n}}\right\}\right]}\right] \\
= & \left(V_{0}^{\tau}-F_{0}\right)\left(E \left[\left(m \frac{S_{t_{1}}}{S_{0}}-(m-1) e^{r \frac{T}{n}}\right) 1_{\left.\left.\left\{\frac{S_{t_{1}}}{S_{0}} \geq \frac{m-1}{m} e^{r \frac{T}{n}}\right\}\right]\right)^{i} .}\right.\right.
\end{aligned}
$$

Finally, notice that the last expectation matches the definition of $E_{1}$, i.e. it holds

$$
E_{1}=\frac{m}{S_{0}} e^{\mu \frac{T}{n}} E\left[e^{-\mu \frac{T}{n}}\left(S_{t_{1}}-\frac{m-1}{m} S_{0} e^{r \frac{T}{n}}\right)^{+}\right]=m e^{\mu \frac{T}{n}} \mathcal{N}\left(d_{1}\right)-e^{r \frac{T}{n}}(m-1) \mathcal{N}\left(d_{2}\right) .
$$

Lemma A.2. Let $E_{1}$ and $E_{2}$ be defined as in Proposition 4.5, then it holds

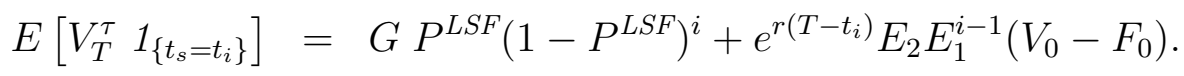

Proof: With Proposition 3.2 and Lemma 4.2 it follows

$$
\begin{aligned}
E\left[V_{T}^{\tau} 1_{\left\{t_{s}=t_{i}\right\}}\right] & =E\left[e^{r\left(T-t_{i}\right)} V_{t_{i}}^{\tau} 1_{\left\{V_{t_{i}}^{\tau} \leq F_{t_{i}}\right\}} \prod_{j=1}^{i-1} 1_{\left\{V_{t_{j}}^{\tau}>F_{t_{j}}\right\}}\right] \\
& =e^{r\left(T-t_{i}\right)} E\left[V_{t_{i}}^{\tau}\left\{\frac{S_{t_{i}}}{\left.S_{t_{i-1}} \leq \frac{m-1}{m} e^{r \frac{T}{n}}\right\}} \prod_{j=1}^{i-1} 1^{i}\left\{\frac{S_{t_{j}}}{S_{t_{j-1}}}>\frac{m-1}{m} e^{r \frac{T}{n}}\right\}\right] .\right.
\end{aligned}
$$


Notice that if there is no shortfall until $t_{i-1}$ it holds, c.f. Proposition 3.2

$$
V_{t_{i}}^{\tau}=F_{t_{i}}+\left(V_{t_{i-1}}^{\tau}-F_{t_{i-1}}\right)\left(m \frac{S_{t_{i}}}{S_{t_{i-1}}}-(m-1) e^{r \frac{T}{n}}\right)
$$

such that

$$
\begin{gathered}
E\left[V_{T}^{\tau} 1_{\left\{t_{s}=t_{i}\right\}}\right]=e^{r\left(T-t_{i}\right)} E\left[F_{t_{i}} 1_{\left\{\frac{S_{t_{i}}}{S_{t_{i-1}}} \leq \frac{m-1}{m} e^{r \frac{T}{n}}\right\}} \prod_{j=1}^{i-1} 1_{\left.\left\{\frac{S_{t_{j}}}{S_{t_{j-1}}}>\frac{m-1}{m} e^{r \frac{T}{n}}\right\}\right]+}\right] \\
e^{r\left(T-t_{i}\right)} E\left[\left(V_{t_{i-1}}^{\tau}-F_{t_{i-1}}\right)\left(m \frac{S_{t_{i}}}{S_{t_{i-1}}}-(m-1) e^{r \frac{T}{n}}\right) 1_{\left\{\frac{S_{t_{i}}}{S_{t_{i-1}}} \leq \frac{m-1}{m} e^{r \frac{T}{n}}\right\}} \prod_{j=1}^{i-1} 1_{\left\{\frac{S_{t_{j}}}{S_{t_{j-1}}}>\frac{m-1}{m} e^{r \frac{T}{n}}\right\}}\right\} .
\end{gathered}
$$

With Lemma A.1 and the assumption that the asset price increments are iid, it follows

$$
\begin{array}{cc}
E\left[V_{T}^{\tau} 1_{\left\{t_{s}=t_{i}\right\}}\right]= & e^{r\left(T-t_{i}\right)} F_{t_{i}} P^{\mathrm{LSF}}\left(1-P^{\mathrm{LSF}}\right)^{i-1} \\
& +e^{r\left(T-t_{i}\right)}\left(V_{0}-F_{0}\right) E_{1}^{i-1} E\left[\left(m \frac{S_{t_{1}}}{S_{0}}-(m-1) e^{r \frac{T}{n}}\right) 1_{\left\{\frac{S_{t_{1}}}{S_{0}} \leq \frac{m-1}{m} e^{r \frac{T}{n}}\right\}}\right] .
\end{array}
$$

Finally, it is straightforward to check that the above expectation satisfies the definition of $E_{2}$.

\section{Appendix B. VARiance of Discrete-Time CPPI}

Analogously to Appendix A, we consider the following two lemmas.

Lemma B.1. Let $\widetilde{E}_{1}$ be defined as in Proposition 4.7, then it holds

$$
E\left[\left(V_{t_{i}}^{\tau}-F_{t_{i}}\right)^{2} \prod_{j=1}^{i} 1\left\{\frac{s_{t_{j}}}{S_{t_{j-1}}}>\frac{m-1}{m} e^{r \frac{T}{n}}\right\}\right]=\left(V_{0}-F_{0}\right)^{2}\left(\widetilde{E}_{1}\right)^{i} \quad \text { for all } i=1, \ldots, n \text {. }
$$

Proof: Analogously to the proof of Lemma A.1 it is straightforward to show that

$$
\begin{aligned}
& E\left[\left(V_{t_{i}}^{\tau}-F_{t_{i}}\right)^{2} \prod_{j=1}^{i}\left\{\frac{S_{t_{j}}}{S_{t_{j-1}}}>\frac{m-1}{m} e^{r \frac{T}{n}}\right\}\right] \\
= & \left(V_{0}-F_{0}\right)^{2}\left(E\left[\left(m \frac{S_{t_{1}}}{S_{0}}-(m-1) e^{r \frac{T}{n}}\right)^{2} 1_{\left\{\frac{S_{t_{1}}}{S_{0}}>\frac{m-1}{m} e^{r \frac{T}{n}}\right\}}\right]\right)^{i}
\end{aligned}
$$

Lemma B.2. Let $\widetilde{E}_{1}$ and $\widetilde{E}_{2}$ be defined as in Proposition 4.7, then it holds

$$
E\left[\left(V_{T}^{\tau}-F_{T}\right)^{2} 1_{\left\{t_{s}=t_{i}\right\}}\right]=e^{2 r\left(T-t_{i}\right)}\left(V_{0}-F_{0}\right)^{2} \widetilde{E}_{2} \widetilde{E}_{1}^{i-1} .
$$

Analogously to the proof of Lemma A.1, observe that

$$
E\left[\left(V_{T}^{\tau}-F_{T}\right)^{2} 1_{\left\{t_{s}=t_{i}\right\}}\right]=e^{2 r\left(T-t_{i}\right)} E\left[\left(V_{t_{i}}^{\tau}-F_{t_{i}}\right)^{2} 1_{\left\{t_{s}=t_{i}\right\}}\right] .
$$


An application of Lemma B.1 gives

$$
\begin{aligned}
& E\left[\left(V_{t_{i}}^{\tau}-F_{t_{i}}\right)^{2} 1_{\left\{t_{s}=t_{i}\right\}}\right]=E\left[\left(V_{t_{i}}^{\tau}-F_{t_{i}}\right)^{2} 1\left\{\frac{S_{t_{i}}}{S_{t_{i-1}}} \leq \frac{m-1}{m} e^{r \frac{T}{n}}\right\} \prod_{j=1}^{i-1} 1_{\left.\left\{\frac{S_{t_{j}}}{S_{t_{j-1}}}>\frac{m-1}{m} e^{r \frac{T}{n}}\right\}\right]}\right] \\
= & E\left[\left(V_{t_{i-1}}^{\tau}-F_{t_{i-1}}\right)^{2}\left(m \frac{S_{t_{i}}}{S_{t_{i-1}}}-(m-1) e^{r \frac{T}{n}}\right)^{2} 1_{\left\{\frac{S_{t_{i}}}{S_{t_{i-1}}} \leq \frac{m-1}{m} e^{r \frac{T}{n}}\right\} \prod_{j=1}^{i-1} 1}\left\{\frac{S_{t_{j}}}{S_{t_{j-1}}}>\frac{m-1}{m} e^{r \frac{T}{n}}\right\}\right] \\
= & E\left[\left(m \frac{S_{t_{1}}}{S_{0}}-(m-1) e^{r \frac{T}{n}}\right)^{2} 1_{\left.\left\{\frac{S_{t_{1}}}{S_{0}} \leq \frac{m-1}{m} e^{r \frac{T}{n}}\right\}\right]\left(V_{0}-F_{0}\right)^{2} \widetilde{E}_{1}^{i-1} .}\right.
\end{aligned}
$$

Finally, it is straightforward to check that the above expectation satisfies the definition of $\widetilde{E}_{2}$.

\section{Appendix C. Convergence (Proof of Proposition 4.8)}

First, we consider the convergence of the shortfall probability, the expected value and the variance of the value.

Lemma C.1. It holds

$$
\lim _{n \rightarrow \infty} P^{S F}=0,
$$

i.e. the shortfall probability converges to zero if the trading restrictions vanish.

Proof: Let $f \in \mathcal{C}^{1}(\mathbb{R})$ such that $\lim _{x \rightarrow \infty} f(x)=1$. With

$$
\lim _{x \rightarrow \infty}(f(x))^{x}=\lim _{x \rightarrow \infty}\left(1+\frac{x(f(x)-1)}{x}\right)^{x}=e^{\lim _{x \rightarrow \infty} x(f(x)-1)}
$$

together with an application of L'Hôpital's rule, i.e.

$$
\lim _{x \rightarrow \infty} x(f(x)-1)=\lim _{x \rightarrow \infty} \frac{f(x)-1}{x^{-1}}=\lim _{x \rightarrow \infty}-x^{2} \frac{\partial f}{\partial x}(x),
$$

it follows $\lim _{x \rightarrow \infty}(f(x))^{x}=e^{\lim _{x \rightarrow \infty}-x^{2} \frac{\partial f}{\partial x}(x)}$ if $\lim _{x \rightarrow \infty}-x^{2} \frac{\partial f}{\partial x}(x)$ exists. Thus,

$$
\begin{aligned}
\lim _{n \rightarrow \infty} P^{S F} & =\lim _{n \rightarrow \infty} 1-\left(1-P^{L S F}\right)^{n}=1-\lim _{n \rightarrow \infty} \mathcal{N}\left(d_{2}\right)^{n} \\
& =1-e^{\lim _{n \rightarrow \infty}-n^{2} \frac{\partial \mathcal{N}\left(d_{2}\right)}{\partial n}}=1-e^{\lim _{n \rightarrow \infty}-n^{2} \mathcal{N}^{\prime}\left(d_{2}\right) \frac{\partial d_{2}}{\partial n}} .
\end{aligned}
$$

The rest of the proof follows immediately with the definition of $d_{2}$, c.f. Proposition 4.5, and

$$
\lim _{n \rightarrow \infty} e^{-n} n^{k}=0 \text { for all } k \in \mathbb{N} \text {. }
$$

Lemma C.2. It holds

$$
\lim _{n \rightarrow \infty} E\left[V_{T}^{\tau}\right]=G+\left(V_{0}-F_{0}\right) e^{(r+m(\mu-r)) T},
$$

i.e. the expected value of the discrete-time CPPI converges to the expected value of a simple CPPI if the trading restrictions vanish. 
Proof: According to Proposition 4.5 it holds

$$
E\left[V_{T}^{\tau}\right]=G+\left(V_{0}-F_{0}\right) E_{1}^{n}+\left(V_{0}-F_{0}\right) e^{-r \frac{T}{n}} E_{2} \frac{e^{r T}-E_{1}^{n}}{1-E_{1} e^{-r \frac{T}{n}}} .
$$

First, we consider the limit of $E_{1}^{n}$. Using the definition of $E_{1}$, c.f. Proposition 4.5, it is straightforward to show that $\lim _{n \rightarrow \infty} E_{1}=1$. According to the proof of Lemma C.1 it holds

$$
\lim _{n \rightarrow \infty} E_{1}^{n}=e^{\lim _{n \rightarrow \infty}-n^{2} \frac{\partial E_{1}}{\partial n}}
$$

where

$$
\begin{aligned}
-n^{2} \frac{\partial E_{1}}{\partial n}= & m \mu T e^{\mu \frac{T}{n}} \mathcal{N}\left(d_{1}\right)-(m-1) r T e^{r \frac{T}{n}} \mathcal{N}\left(d_{2}\right) \\
& -m e^{\mu \frac{T}{n}} n^{2} \mathcal{N}^{\prime}\left(d_{1}\right) \frac{\partial d_{1}}{\partial n}+(m-1) e^{r \frac{T}{n}} n^{2} \mathcal{N}^{\prime}\left(d_{2}\right) \frac{\partial d_{2}}{\partial n} .
\end{aligned}
$$

Notice that the last two terms on the right-hand side vanish for $n \rightarrow \infty$. Besides, with the definitions of $d_{1}$ and $d_{2}$, c.f. Proposition 4.5, it immediately follows

$$
\lim _{n \rightarrow \infty} \mathcal{N}\left(d_{1}\right)=1, \quad \lim _{n \rightarrow \infty} \mathcal{N}\left(d_{2}\right)=1
$$

such that

$$
\lim _{n \rightarrow \infty}\left(V_{0}-F_{0}\right) E_{1}^{n}=\left(V_{0}-F_{0}\right) e^{m \mu T-(m-1) r T} .
$$

Thus, it is still to show that the last term of the right hand side of Equation (8) converges to zero. Inserting $E_{2}$ according to its definition, c.f. Proposition 4.5, the relevant term is

$$
\left(V_{0}-F_{0}\right)\left(e^{r T}-E_{1}^{n}\right)\left(1+\frac{m\left(e^{(\mu-r) \frac{T}{n}}-1\right)}{1-E_{1} e^{-r \frac{T}{n}}}\right) .
$$

Notice that $\lim _{n \rightarrow \infty} m\left(e^{(\mu-r) \frac{T}{n}}-1\right)=0$ and $\lim _{n \rightarrow \infty} 1-E_{1} e^{-r \frac{T}{n}}=0$. With the rule of L'Hôpital and similar arguments as above it follows

$$
\begin{aligned}
\lim _{n \rightarrow \infty} \frac{m\left(e^{(\mu-r) \frac{T}{n}}-1\right)}{1-E_{1} e^{-r \frac{T}{n}}} & =\lim _{n \rightarrow \infty}\left(\frac{-\frac{m(\mu-r) T}{n^{2}} e^{(\mu-r) \frac{T}{n}}}{-\frac{E_{1} r T e^{-r \frac{T}{n}}}{n^{2}}-e^{-r \frac{T}{n}} \frac{\partial E_{1}}{\partial n}}\right) \\
& =\frac{m(\mu-r) T}{r T-(m \mu T-(m-1) r T)}=-1 .
\end{aligned}
$$

LEMMA C.3. It holds

$$
\lim _{n \rightarrow \infty} \operatorname{Var}\left[V_{T}^{\tau}\right]=\left(V_{0}-F_{0}\right)^{2} e^{2(r+m(\mu-r)) T}\left(e^{m^{2} \sigma^{2} T}-1\right),
$$

i.e. the variance of the discrete-time CPPI converges to the variance of a simple CPPI if the trading restrictions vanish.

Proof: Recall that according to Proposition 4.7 it holds

$$
\operatorname{Var}\left[V_{T}^{\tau}\right]=\left(V_{0}-F_{0}\right)^{2} \widetilde{E}_{1}^{n}+\left(V_{0}-F_{0}\right)^{2} e^{-2 r \frac{T}{n}} \widetilde{E}_{2} \frac{e^{2 r T}-\widetilde{E}_{1}^{n}}{1-e^{-2 r \frac{T}{n}} \tilde{E}_{1}}-\left(E\left[V_{T}^{\tau}\right]-G\right)^{2} .
$$


Analogously to the proof of Lemma C.2, it can be shown that $\widetilde{E}_{2} \frac{e^{2 r T}-\widetilde{E}_{1}^{n}}{1-e^{-2 r \frac{T}{n}} \widetilde{E}_{1}} \stackrel{n \rightarrow \infty}{\longrightarrow} 0$. For the convergence of $\widetilde{E}_{1}^{n}$ we use again that $\lim _{n \rightarrow \infty} \widetilde{E}_{1}^{n}=e^{\lim _{n \rightarrow \infty}-n^{2} \frac{\partial \widetilde{E}_{1}}{\partial n}}$. Notice that

$$
\begin{aligned}
-n^{2} \frac{\partial \widetilde{E}_{1}}{\partial n}= & m^{2} e^{\left(2 \mu+\sigma^{2}\right) \frac{T}{n}}\left(2 \mu+\sigma^{2}\right) T \mathcal{N}\left(d_{3}\right)-2 m(m-1) e^{(\mu+r) \frac{T}{n}}(\mu+r) T \mathcal{N}\left(d_{1}\right) \\
& +(m-1)^{2} e^{2 r \frac{T}{n}} 2 r T \mathcal{N}\left(d_{2}\right)-m^{2} e^{\left(2 \mu+\sigma^{2}\right) \frac{T}{n}} n^{2} \mathcal{N}^{\prime}\left(d_{3}\right) \frac{\partial d_{3}}{\partial n} \\
& +2 m(m-1) e^{(\mu+r) \frac{T}{n}} n^{2} \mathcal{N}^{\prime}\left(d_{1}\right) \frac{\partial d_{1}}{\partial n}-(m-1)^{2} e^{2 r \frac{T}{n}} n^{2} \mathcal{N}^{\prime}\left(d_{2}\right) \frac{\partial d_{2}}{\partial n}
\end{aligned}
$$

Similar arguments to the ones given in the proofs of Lemma C.2 and Lemma C.1 imply

$$
\lim _{n \rightarrow \infty} \widetilde{E}_{1}^{n}=e^{m^{2}\left(2 \mu+\sigma^{2}\right) T-2 m(m-1)(\mu+r) T+(m-1)^{2} 2 r T}=e^{2(r+m(\mu-r)) T} e^{m^{2} \sigma^{2} T} .
$$

Finally, Lemma C.2 immediately gives

$$
\lim _{n \rightarrow \infty}\left(E\left[V_{T}^{\tau}\right]-G\right)^{2}=\left(V_{0}-F_{0}\right)^{2} e^{2(r+m(\mu-r)) T} .
$$

In order to prove Proposition 4.8 it is remains to show that, for $n \rightarrow \infty$, the limiting distribution of $\ln \left(V_{T}^{\tau}\right)$ is a normal distribution . Let

$$
\zeta_{n}:=\sum_{i=1}^{n} \underbrace{\ln \left(m \frac{S_{t_{i}}}{S_{t_{i+1}}}-(m-1) e^{r \frac{T}{n}}\right)}_{=: \xi_{i, n}} .
$$

With Lemma C.1, i.e. $\lim _{n \rightarrow \infty} P^{S F}=0$, it follows that it is sufficient to show that the limiting distribution of $\zeta_{n}$ is a normal distribution. Applying the results for rowwise independent arrays of Gnedenko and Kolmogorov (1954), c.f. in particular Theorem 1 in Ch. $5 \S 26$, it remains to show that

$$
\sum_{i=1}^{n} P\left(\left|\xi_{i, n}\right|>\epsilon\right) \stackrel{n \rightarrow \infty}{\longrightarrow} 0 \text { for all } \epsilon>0 .
$$

Using the independency, one only needs to show that

$$
n P\left(\left|\xi_{1, n}\right|>\epsilon\right) \stackrel{n \rightarrow \infty}{\longrightarrow} 0 \text { for all } \epsilon>0 .
$$

This proof is straightforward, i.e. it is given by arguments which are similar to the ones used in the proofs of the above lemmas.

\section{Appendix D. ExpeCted terminal VAlue of Discrete-time CPPI IS MONOTONICALLY INCREASING IN THE VOLATILITY}

With Proposition 4.5 and the definition of $E_{2}$ it follows that

$$
E\left[V_{T}^{\tau}\right]=V_{0} e^{r T}+\left(V_{0}-F_{0}\right) m\left(e^{(\mu-r) \frac{T}{n}}-1\right) \frac{e^{r T}-E_{1}^{n}}{1-E_{1} e^{-r \frac{T}{n}}} .
$$

It is straightforward to show that $E_{1}>e^{r \frac{T}{n}}$. For $\mu>r$, the expected terminal value of the discrete CPPI strategy is always larger than the investment in the riskless asset. This 
is quite intuitive.

Now, consider the derivative with respect to $\sigma$, i.e.

$\frac{\partial E\left[V_{T}^{\tau}\right]}{\partial \sigma}=m\left(V_{0}-F_{0}\right)\left(e^{(\mu-r) \frac{T}{n}}-1\right) \frac{-n E_{1}^{n-1} \frac{\partial E_{1}}{\partial \sigma}\left(1-E_{1} e^{-r \frac{T}{n}}\right)-\left(E_{1}^{n}-e^{r T}\right) \frac{\partial E_{1}}{\partial \sigma} e^{-r \frac{T}{n}}}{\left(1-E_{1} e^{-r \frac{T}{n}}\right)^{2}}$.

For $\mu>r$, the leading factors are positive. Besides, we have

$$
\frac{\partial E_{1}}{\partial \sigma}>0
$$

The proof of the above inequality is omitted here. In particular, analogous calculations as for the determination of the so-called vega of a call-option price in a Black/Scholes-type model are needed. Finally, it is to show that

$$
-n E_{1}^{n-1}\left(1-E_{1} e^{-r \frac{T}{n}}\right)-\left(E_{1}^{n}-e^{r T}\right) e^{-r \frac{T}{n}} \geq 0 .
$$

An application of Bernoulli's inequality gives

$$
\begin{aligned}
& n E_{1}^{n-1}\left(E_{1} e^{-r \frac{T}{n}}-1\right)-e^{-r \frac{T}{n}}\left(E_{1}^{n}-e^{r T}\right) \\
= & e^{-r \frac{T}{n}}\left(n E_{1}^{n-1}\left(E_{1}-e^{r \frac{T}{n}}\right)-E_{1}^{n}+E_{1}^{n}\left(1+\frac{e^{r \frac{T}{n}}-E_{1}}{E_{1}}\right)^{n}\right) \\
\geq & e^{-r \frac{T}{n}}\left(n E_{1}^{n-1}\left(E_{1}-e^{r \frac{T}{n}}\right)-E_{1}^{n}+E_{1}^{n}\left(1+n \frac{e^{r \frac{T}{n}}-E_{1}}{E_{1}}\right)\right)=0 .
\end{aligned}
$$

Notice that because $E_{1}>e^{r \frac{T}{n}}$, the above inequality is also strict. 


\section{REFERENCES}

Artzner, P., Delbaen, F., Eber, J., Heath, D., 1999. Coherent measures of risk. Mathematical Finance 9, 203-228.

Avellaneda, M., Levy, A., Parás, A., 1995. Pricing and Hedging Derivative Securities in Markets with Uncertain Volatilities. Applied Mathematical Finance 2, 73-88.

Avellaneda, M., Parás, A., 1994. Dynamic Hedging Portfolios for Derivative Securities in the Presence of Large Transaction Costs. Applied Mathematical Finance 1, 165-193.

Basak, S., 1995. A General Equilibrium Model of Portfolio Insurance. Review of Financial Studies 8, 1059-1090.

Basak, S., 2002. A Comparative Study of Portfolio Insurance. The Journal of Economic Dynamics and Control 26, 1217-1241.

Bensaid, B., Lesne, J., Scheinkman, J., 1992. Derivative Asset Pricing with Transaction Costs. Mathematical Finance 2, 63-86.

Bergman, Y., Grundy, B., Wiener, Z., 1996. General Properties of Option Prices. Journal of Finance 51, 1573-1610.

Bertrand, P., Prigent, J.-L., 2002a. Portfolio Insurance Strategies: OBPI versus CPPI. Discussion paper, GREQAM and Université Montpellier1.

Bertrand, P., Prigent, J.-L., 2002b. Portfolio Insurance: The Extreme Value Approach to the CPPI. Finance 23.

Bertrand, P., Prigent, J.-L., 2003. Portfolio Insurance Strategies: A Comparison of Standard Methods When the Volatility of the Stoch is Stochastic. International Journal of Business 8, 15-31.

Bertsimas, D., Kogan, L., Lo, A., 1998. When is Time Continuous. Discussion paper, Massachusetts Institute of Technology (MIT).

Black, F., Jones, R., 1987. Simplifying Portfolio Insurance. The Journal of Portfolio Management 14, 48-51.

Black, F., Perold, A., 1992. Theory of Constant Proportion Portfolio Insurance. The Journal of Economic Dynamics and Control 16, 403-426.

Black, F., Scholes, M., 1973. The Pricing of Options and Corporate Liabilities. Journal of Political Economics 81, 637-654.

Bookstaber, R., Langsam, J., 2000. Portfolio Insurance Trading Rules. The Journal of Futures Markets 8, 15-31.

Boyle, P., Emanuel, D., 1980. Discretely Adjusted Option Hedges. Journal of Financial Economics 8, 259-282.

Boyle, P., Vorst, T., 1992. Option Replication in Discrete Time with Transaction Costs. Journal of Finance 47, 271-294.

Brennan, M., Schwartz, E., 1989. Portfolio Insurance and Financial Market Equilibrium. Journal of Business 62, 455-472.

Browne, S., 1999. Beating a Moving Target: Optimal Portfolio Strategies for Outperforming a Stochastic Benchmark. Finance and Stochastics 3, 275-294.

Cox, J., Huang, C.-F., 1989. Optimal Consumption and Portfolio Policies when the Asset Price follows a Diffusion Process. Journal of Economic Theory 49, 33-83.

Cvitanic, J., Karatzas, I., 1996. On Portfolio Optimization under Drawdown Constraints. Mathematical Finance 65, 35-45.

Cvitanic, J., Karatzas, I., 1999. On dynamic measures of risk. Finance and Stochastics 3, 451-482. 
Do, B., 2002. Do, B.H.. Accounting and Finance 42, 279-296.

Dudenhausen, A., Schlögl, E., Schlögl, L., 1998. Robustness of Gaussian Hedges under Parameter and Model Misspecification. Discussion paper, University of Bonn, Department of Statistics.

El Karoui, N., Jeanblanc, M., Lacoste, V., 2005. Optimal Portfolio Management with American Capital Guarantee. Journal of Economic Dynamics and Control 29, 449-468.

El Karoui, N., Jeanblanc-Picqué, M., Shreve, S., 1998. Robustness of the Black and Scholes Formula. Mathematical Finance 8, 93-126.

Fletcher, P., 2005. Guaranteed to take the credit. Risk Magazine 18.

Gnedenko, B., Kolmogorov, A., 1954. Limit distributions for sums of independent random variables, Addison-Wesley, New York.

Grannan, E., Swindle, G., 1996. Minimizing Transaction Costs of Option Hedging Strategies. Mathematical Finance 6, 341-364.

Grossman, S., Villa, J., 1989. Portfolio Insurance in Complete Markets: A Note. Journal of Business 62, 473-476.

Grossman, S., Zhou, J., 1993. Optimal Investment Strategies for Controlling Drawdowns. Mathematical Finance 3, 241-276.

Grossman, S., Zhou, J., 1996. Equilibrium Analysis of Portfolio Insurance. Journal of Finance 51, 1379-1403.

Hayashi, T., Mykland, P., 2005. Evaluating Hedging Errors: An Asymptotic Approach. Mathematical Finance 15, 309-343.

Hobson, D., 1998. Volatility Misspecification, Option Pricing and Superreplication via Coupling. Annals of Applied Probability 8, 193-205.

Leland, H., 1985. Option Pricing and Replication with Transaction Costs. Journal of Finance 40, 1283-1301.

Leland, H., Rubinstein, M., 1976. The Evolution of Potfolio Insurance, in: D.L. Luskin, ed., Portfolio Insurance: A guide to Dynamic Hedging, Wiley.

Lyons, T., 1995. Uncertain Volatility and the Risk-free Synthesis of Derivatives. Applied Mathematical Finance 2, 117-133.

Mahayni, A., 2003. Effectiveness of Hedging Strategies under Model Misspecification and Trading Restrictions. International Journal of Theoretical and Applied Finance 6, 521552.

Merton, R., 1971. Optimal Consumption and Portfolio Rules in a Continuous Time Model. Journal of Economic Theory 3, 373-413.

Schweizer, M., 2001. A Guided Tour on Quadratic Hedging Approaches, in: Jouini, E., Cvitanic, J., Musiela, M. (Eds.), Option Pricing, Interest Rates and Risk Management, Cambridge University Press, pp. 538-574.

Talay, D., Zheng, Z., 2003. Quantiles of the Euler Scheme for Diffusion Processes anf Financial Applications. Mathematical Finance 13, 187-199.

Tepla, L., 2000. Optimal portfolio policies with borrowing and shortsale constraints. The Journal of Economic Dynamics and Control 24, 1623-1639.

Tepla, L., 2001. Optimal Investment with Minimum Performance Constraints. The Journal of Economic Dynamics and Control 25, 1629-1645.

Toft, K. B., 1996. On the Mean-Variance Tradeoff in Option Replication with Transaction Costs. Journal of Financial and Quantitative Analysis 31, 233-263. 